\title{
Ruin by Dynamic Contagion Claims
}

\author{
Angelos Dassios*, Hongbiao Zhao \\ Department of Statistics, London School of Economics, Houghton Street, London WC2A 2AE, United Kingdom
}

\begin{abstract}
In this paper, we consider a risk process with the arrival of claims modelled by a dynamic contagion process, a generalisation of the Cox process and Hawkes process introduced by Dassios and Zhao (2011). We derive results for the infinite horizon model that are generalisations of the Cramér-Lundberg approximation, Lundberg's fundamental equation, some asymptotics as well as bounds for the probability of ruin. Special attention is given to the case of exponential jumps and two numerical examples are provided.
\end{abstract}

Keywords: Dynamic contagion process, Ruin probability, Generalised Lundberg's fundamental equation, Cramér-Lundberg approximation, Change of measure, Martingale method

2010 MSC: Primary: 91B30, Secondary: 60J25, 60J75, 60J85

JEL: C10

\section{Introduction}

In the classical Cramér-Lundberg risk model, the arrival of claims is modelled by a Poisson process. As substantially discussed in the literature, this model is often not realistic in practice and hence a variety of extensions have been studied. Many researchers, such as Björk and Grandell (1988), Embrechts, Grandell and Schmidli (1993) had already suggested use the Cox process to model the arrival of claims, see also the book by Grandel (1991). Schmidli (1996) investigated the case for a Cox process with a piecewise constant intensity. More recently, Albrecher and Asmussen (2006) discussed a Cox process with shot noise intensity. On the other hand, only a few researchers have proposed risk models using self-excited processes, due to the observation of the clustering arrival of claims in reality, a similar pattern in the credit risk from the financial market, particularly during the current economic crisis. Stabile and Torrisi (2010) looked at the ruin problem in a model using the Hawkes process, a self-excited point process introduced by Hawkes (1971).

To capture the clustering phenomenon as well as some common external factors involved for the arrival of claims within one single consistent framework, in this paper, we extend further to use the dynamic contagion process introduced by Dassios and Zhao (2011), a generalisation of the externally excited Cox process with shot noise intensity (with exponential decay) and the self-excited Hawkes process (with exponential decay). It could be particularly useful for modelling the dependence structure of the underlying arriving events with dynamic contagion impact

${ }^{*}$ Corresponding Author: Angelos Dassios (Email: A.Dassios@1se.ac.uk) 
from both endogenous and exogenous factors. In this paper, we try to generalise results obtained for the classical model.

We organise our paper as follows. Section 2 provides distributional results we will use, mainly developed in Dassios and Zhao (2011). Section 3 formulates the problem. It also provides numerical examples and some asymptotics that are based on simulations. In Section 4, we use the martingale method and generalise Lundberg's fundamental equation. We derive bounds for the ruin probability in Section 5. In Section 6, we derive all results via a change of measure. This makes simulations more efficient as ruin is certain under the new measure. Section 7 concentrates on exponentially distributed claims. Our results are illustrated by two numerical examples.

\section{Dynamic Contagion Process}

The dynamic contagion process includes both the self-excited jumps (which are distributed according to the branching structure of a Hawkes process with exponential fertility rate) and the externally excited jumps (which are distributed according to a particular shot noise Cox process). We directly use the definition of the dynamic contagion process from Dassios and Zhao (2011).

Definition 2.1 (Dynamic Contagion Process). The dynamic contagion process is a cluster point process $\mathbb{D}$ on $\mathbb{R}_{+}$: The number of points in the time interval $(0, t]$ is defined by $N_{t}=N_{\mathbb{D}(0, t]}$. The cluster centers of $\mathbb{D}$ are the particular points called immigrants, and the other points are called offspring. They have the following structure:

(a) The immigrants are distributed according to a Cox process $A$ with points $\left\{D_{m}\right\}_{m=1,2, \ldots} \in(0, \infty)$ and shot noise stochastic intensity process

$$
a+\left(\lambda_{0}-a\right) e^{-\delta t}+\sum_{i \geq 1} Y_{i}^{(1)} e^{-\delta\left(t-T_{i}^{(1)}\right)} \mathbb{I}\left\{T_{i}^{(1)} \leq t\right\},
$$

where

- $a \geq 0$ is the constant reversion level;

- $\lambda_{0}>0$ is a constant as the initial value of the stochastic intensity process (defined later by (1));

- $\delta>0$ is the constant rate of exponential decay;

- $\left\{Y_{i}^{(1)}\right\}_{i=1,2 \ldots . . .}$ is a sequence of independent identical distributed positive (externally excited) jumps with distribution function $H(y), y>0$, at the corresponding random times $\left\{T_{i}^{(1)}\right\}_{i=1,2, \ldots}$ following a homogeneous Poisson process $M_{t}$ with constant intensity $\rho>0$;

- II is the indicator function.

(b) Each immigrant $D_{m}$ generates a cluster $C_{m}=C_{D_{m}}$, which is the random set formed by the points of generations $0,1,2, \ldots$ with the following branching structure:

the immigrant $D_{m}$ is said to be of generation 0. Given generations $0,1, \ldots, j$ in $C_{m}$, each point $T^{(2)} \in C_{m}$ of generation $j$ generates a Cox process on $\left(T^{(2)}, \infty\right)$ of offspring of generation $j+1$ with the stochastic intensity $Y^{(2)} e^{-\delta\left(-T^{(2)}\right)}$ where $Y^{(2)}$ is a positive (self-excited) jump at time $T^{(2)}$ with distribution function $G(y), y>0$, independent of the points of generation $0,1, \ldots, j$. 
(c) Given the immigrants, the centered clusters

$$
C_{m}-D_{m}=\left\{T^{(2)}-D_{m}: T^{(2)} \in C_{m}\right\}, \quad D_{m} \in A,
$$

are independent identical distributed, and independent of $A$.

(d) $\mathbb{D}$ consists of the union of all clusters, i.e.

$$
\mathbb{D}=\bigcup_{m=1,2, \ldots} C_{D_{m}}
$$

Therefore, the dynamic contagion process can also be defined as a point process $N_{t} \equiv$ $\left\{T_{k}^{(2)}\right\}_{k \geq 1}$ on $\mathbb{R}_{+}$, with the non-negative $\mathcal{F}_{t}-$ stochastic intensity process $\lambda_{t}$ following the piecewise deterministic dynamics with positive jumps, i.e.

$$
\lambda_{t}=a+\left(\lambda_{0}-a\right) e^{-\delta t}+\sum_{i \geq 1} Y_{i}^{(1)} e^{-\delta\left(t-T_{i}^{(1)}\right)} \mathbb{I}\left\{T_{i}^{(1)} \leq t\right\}+\sum_{k \geq 1} Y_{k}^{(2)} e^{-\delta\left(t-T_{k}^{(2)}\right)} \mathbb{I}\left\{T_{k}^{(2)} \leq t\right\},
$$

where

- $\left\{\mathcal{F}_{t}\right\}_{t \geq 0}$ is a history of the process $N_{t}$, with respect to which $\left\{\lambda_{t}\right\}_{t \geq 0}$ is adapted,

- $\left\{Y_{k}^{(2)}\right\}_{k=1,2, \ldots}$ is a sequence of independent identical distributed positive (self-excited) jumps with distribution function $G(y), y>0$, at the corresponding random times $\left\{T_{k}^{(2)}\right\}_{k=1,2, \ldots}$,

- the sequences $\left\{Y_{i}^{(1)}\right\}_{i=1,2, \ldots},\left\{T_{i}^{(1)}\right\}_{i=1,2, \ldots}$ and $\left\{Y_{k}^{(2)}\right\}_{k=1,2, \ldots}$ are assumed to be independent of each other.

With the aid of the piecewise deterministic Markov process theory and using the results in Davis (1984), the infinitesimal generator of the dynamic contagion process $\left(\lambda_{t}, N_{t}, t\right)$ acting on a function $f(\lambda, n, t) \in \Omega(\mathcal{A})$ is given by

$$
\begin{aligned}
\mathcal{A} f(\lambda, n, t)= & \frac{\partial f}{\partial t}-\delta(\lambda-a) \frac{\partial f}{\partial \lambda}+\rho\left(\int_{0}^{\infty} f(\lambda+y, n, t) \mathrm{d} H(y)-f(\lambda, n, t)\right) \\
& +\lambda\left(\int_{0}^{\infty} f(\lambda+y, n+1, t) \mathrm{d} G(y)-f(\lambda, n, t)\right),
\end{aligned}
$$

where $\Omega(\mathcal{A})$ is the domain of generator $\mathcal{A}$ such that $f(\lambda, n, t)$ is differentiable with respect to $\lambda, t$ for all $\lambda, n$ and $t$, and

$$
\begin{aligned}
\left|\int_{0}^{\infty} f(\lambda+y, n, t) \mathrm{d} H(y)-f(\lambda, n, t)\right| & <\infty, \\
\left|\int_{0}^{\infty} f(\lambda+y, n+1, t) \mathrm{d} G(y)-f(\lambda, n, t)\right| & <\infty .
\end{aligned}
$$

To give an intuitive picture of this new process by stochastic intensity representation, we present Figure 1 for illustrating how the externally excited jumps $\left\{Y_{i}^{(1)}\right\}_{i=1,2, \ldots}$ (marked by single arrow $\downarrow$ ) and self-excited jumps $\left\{Y_{k}^{(2)}\right\}_{k=1,2, \ldots}$ (marked by double arrow $\uparrow$ ) in the intensity process $\lambda_{t}$ interact with the point process $N_{t}$. 


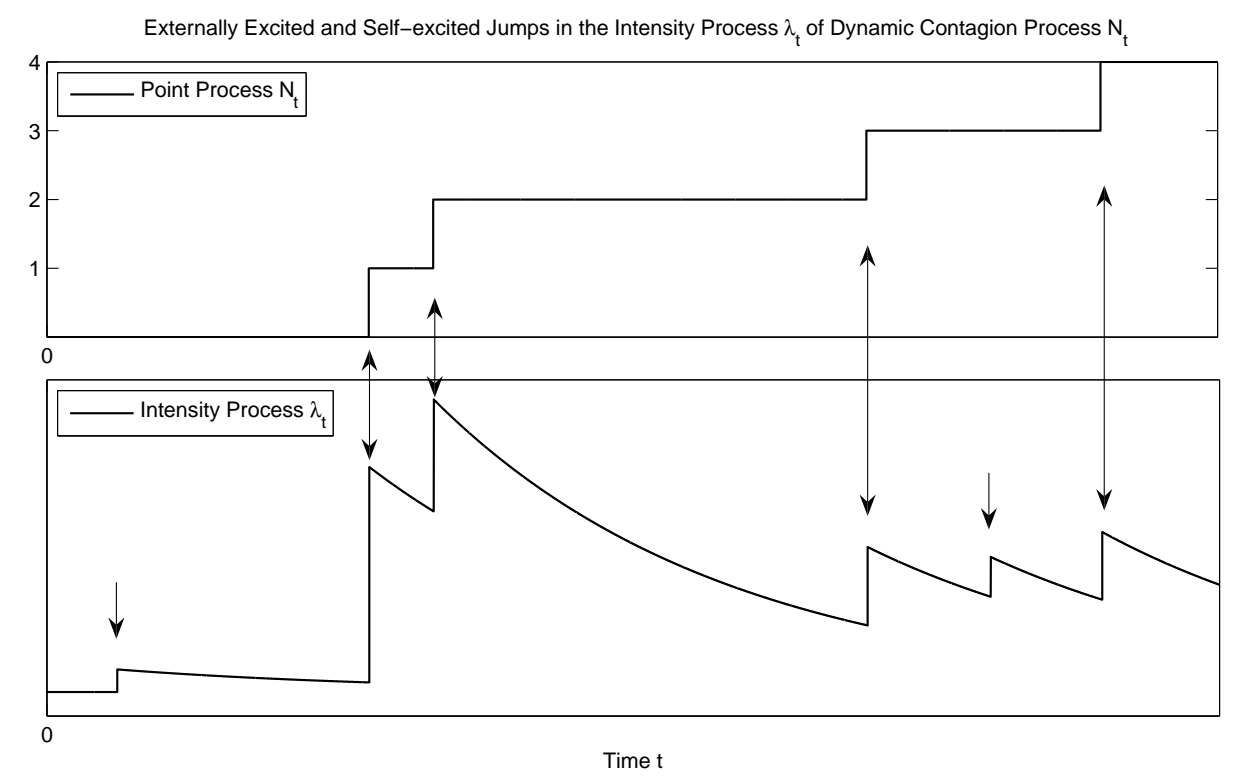

Figure 1: Externally Excited and Self-excited Jumps in Intensity Process $\lambda_{t}$ of A Dynamic Contagion Process

The dynamic contagion process has some key distributional properties which will be used in this paper and are listed as below. The corresponding proofs have been given by Dassios and Zhao (2011) and we omit them here.

Proposition 2.1. $\delta>\mu_{1_{G}}$ is the stationarity condition of the intensity process $\lambda_{t}$ of a dynamic contagion process, where

$$
\mu_{1_{G}}=: \int_{0}^{\infty} y \mathrm{~d} G(y) .
$$

Theorem 2.1. If $\delta>\mu_{1_{G}}$, then the Laplace transform of the asymptotic distribution of $\lambda_{t}$ is given by

$$
\hat{\Pi}(v)=: \lim _{t \rightarrow \infty} \mathbb{E}\left[e^{-v \lambda_{t}} \mid \lambda_{0}\right]=\exp \left(-\int_{0}^{v} \frac{a \delta u+\rho[1-\hat{h}(u)]}{\delta u+\hat{g}(u)-1} \mathrm{~d} u\right),
$$

and (2) is also the Laplace transform of the stationary distribution of the process $\left\{\lambda_{t}\right\}_{t \geq 0}$, where

$$
\hat{h}(u)=: \int_{0}^{\infty} e^{-u y} \mathrm{~d} H(y), \quad \hat{g}(u)=: \int_{0}^{\infty} e^{-u y} \mathrm{~d} G(y) .
$$

Corollary 2.1. If $\delta>\mu_{1_{G}}$, then,

$$
\lim _{t \rightarrow \infty} \mathbb{E}\left[\lambda_{t} \mid \lambda_{0}\right]=\frac{\mu_{1_{H}} \rho+a \delta}{\delta-\mu_{1_{G}}},
$$

where

$$
\mu_{1_{H}}=: \int_{0}^{\infty} y \mathrm{~d} H(y),
$$


and (3) is also the mean of stationary distribution of the process $\left\{\lambda_{t}\right\}_{t \geq 0}$.

Theorem 2.2. For any function $f \in \Omega(\mathcal{A})$, we have

$$
\int_{E} \mathcal{A} f(\lambda) \Pi(\lambda) \mathrm{d} \lambda=0,
$$

where $E=[a, \infty)$ is the domain of $\lambda, \mathcal{A} f(\lambda)$ is the infinitesimal generator of the dynamic contagion process acting on $f(\lambda)$, i.e.

$$
\mathcal{A} f(\lambda)=-\delta(\lambda-a) \frac{\mathrm{d} f(\lambda)}{\mathrm{d} \lambda}+\rho\left(\int_{0}^{\infty} f(\lambda+y) \mathrm{d} H(y)-f(\lambda)\right)+\lambda\left(\int_{0}^{\infty} f(\lambda+z) \mathrm{d} G(z)-f(\lambda)\right),
$$

and $\Pi(\lambda)$ is the density function of $\lambda$ with the Laplace transform specified by (2).

Theorem 2.3. If the externally excited and self-excited jumps follow exponential distributions, i.e. $H \sim \operatorname{Exp}(\alpha), G \sim \operatorname{Exp}(\beta)$ and $\delta \beta>1$, then, the stationary distribution of $\lambda_{t}$ is given by

$$
\begin{cases}a+\Gamma_{1}+\Gamma_{2} & \text { for } \alpha \geq \beta \\ a+\Gamma_{3}+\tilde{B} & \text { for } \alpha<\beta \text { and } \alpha \neq \beta-\frac{1}{\delta} \\ a+\Gamma_{4}+\tilde{P} & \text { for } \alpha=\beta-\frac{1}{\delta}\end{cases}
$$

where independent random variables

$$
\begin{aligned}
\Gamma_{1} \sim \operatorname{Gamma}\left(\frac{1}{\delta}\left(a+\frac{\rho}{\delta(\alpha-\beta)+1}\right), \frac{\delta \beta-1}{\delta}\right) ; \\
\Gamma_{2} \sim \operatorname{Gamma}\left(\frac{\rho(\alpha-\beta)}{\delta(\alpha-\beta)+1}, \alpha\right) ; \\
\Gamma_{3} \sim \operatorname{Gamma}\left(\frac{a+\rho}{\delta}, \frac{\delta \beta-1}{\delta}\right) ; \\
\Gamma_{4} \sim \operatorname{Gamma}\left(\frac{a+\rho}{\delta}, \alpha\right) ; \\
\tilde{B} \stackrel{\mathcal{D}}{=} \sum_{i=1}^{N_{1}} X_{i}^{(1)}, N_{1} \sim \operatorname{NegBin}\left(\frac{\rho}{\delta} \frac{\beta-\alpha}{\gamma_{1}-\gamma_{2}}, \frac{\gamma_{2}}{\gamma_{1}}\right), X_{i}^{(1)} \sim \operatorname{Exp}\left(\gamma_{1}\right), \\
\quad \gamma_{1}=\max \left\{\alpha, \frac{\delta \beta-1}{\delta}\right\}, \gamma_{2}=\min \left\{\alpha, \frac{\delta \beta-1}{\delta}\right\} ; \\
\tilde{P} \quad \stackrel{D}{=} \sum_{i=1}^{N_{2}} X_{i}^{(2)}, N_{2} \sim \operatorname{Poisson}\left(\frac{\rho}{\delta^{2} \alpha}\right), X_{i}^{(2)} \sim \operatorname{Exp}(\alpha) .
\end{aligned}
$$

Remark 2.1. $\tilde{B}$ follows a compound negative binomial distribution with underlying exponential jumps, and $\tilde{P}$ follows a compound Poisson distribution with underlying exponential jumps. Theorem 2.3 implies that the Laplace transform of $\lambda_{t}$ is given by

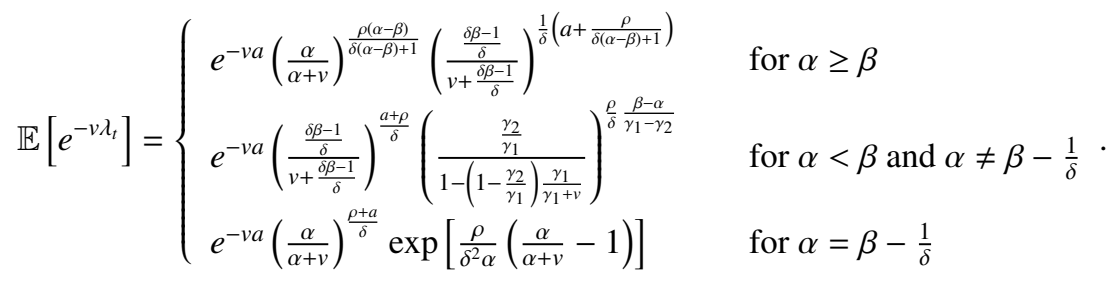




\section{Ruin Problem}

We consider a company with its surplus process $X_{t}$ in continuous time on a probability space $(\Omega, \mathcal{F}, \mathbb{P})$,

$$
X_{t}=x+c t-\sum_{i=1}^{N_{t}} Z_{i}, \quad t \geq 0,
$$

where

- $X_{0}=x \geq 0$ is the initial reserve at time $t=0$;

- $c>0$ is the constant rate of premium payment per time unit;

- $N_{t}$ is a point process $\left(N_{0}=0\right)$ counting the number of cumulative arrived claims in the time interval $(0, t]$, driven by a dynamic contagion process with its stochastic intensity process $\lambda_{t}$ and the initial intensity $\lambda_{0}=\lambda>0$;

- $\left\{Z_{i}\right\}_{i=1,2, \ldots}$ is a sequence of independent identical distributional positive random variables (claim sizes) with distribution function $Z(z), z>0$, and also independent of $N_{t}$; the mean, Laplace transform of density function and tail are denoted respectively by

$$
\mu_{1_{Z}}=: \int_{0}^{\infty} z \mathrm{~d} Z(z), \quad \hat{z}(u)=: \int_{0}^{\infty} e^{-u z} \mathrm{~d} Z(z), \quad \bar{Z}(x)=: \int_{x}^{\infty} \mathrm{d} Z(s) .
$$

The surplus process $X_{t}$ is a right-continuous function of time $t$.

Definition 3.1 (Ruin Time). The ruin (stopping) time $\tau^{*}$ is defined by

$$
\tau^{*}=:\left\{\begin{array}{l}
\inf \left\{t>0 \mid X_{t} \leq 0\right\} \\
\inf \{\varnothing\}=\infty
\end{array} \quad \text { if } X_{t}>0 \text { for all } t ;\right.
$$

in particular, $\tau^{*}=\infty$ means ruin does not occur.

We are interested in the ruin probability in finite time,

$$
P\left\{\tau^{*}<t \mid X_{0}=x, \lambda_{0}=\lambda\right\}
$$

in particular, the ultimate ruin probability in infinite time,

$$
P\left\{\tau^{*}<\infty \mid X_{0}=x, \lambda_{0}=\lambda\right\}=: \lim _{t \rightarrow \infty} P\left\{\tau^{*}<t \mid X_{0}=x, \lambda_{0}=\lambda\right\} ;
$$

and also the ultimate ruin probability in infinite time when the intensity process $\lambda_{t}$ has stationary distribution,

$$
P\left\{\tau^{*}<\infty \mid X_{0}=x, \lambda_{0} \sim \Pi\right\},
$$

where $\Pi$ is the stationary distribution of $\lambda_{t}$ given by Theorem 2.1 . 


\subsection{Net Profit Condition}

Theorem 3.1. If $\delta>\mu_{1_{G}}$ and the arrival of claims is driven by a dynamic contagion process, then, the net profit condition is given by

$$
c>\frac{\mu_{1_{H}} \rho+a \delta}{\delta-\mu_{1_{G}}} \mu_{1_{Z}} .
$$

Proof. Obviously, we have the expectation of surplus process $X_{t}$ defined by (5),

$$
\mathbb{E}\left[X_{t}\right]=x+c t-\mu_{1 z} \mathbb{E}\left[\lambda_{t}\right] t .
$$

If $\delta>\mu_{1_{G}}$ and the net profit condition holds, by Corollary 2.1, we have

$$
\lim _{t \rightarrow \infty} \frac{\mathbb{E}\left[X_{t}\right]}{t}=c-\frac{\mu_{1_{H}} \rho+a \delta}{\delta-\mu_{1_{G}}} \mu_{1_{Z}}>0 .
$$

\subsection{Simulation Examples}

Before giving mathematical proofs, we can have a first glance at this ruin problem via Monte Carlo simulation. Assume the stationarity condition for $\lambda_{t}$ and net profit condition for $X_{t}$ both hold, and the two types of jump sizes and claim sizes all follow exponential distributions, i.e. $H \sim \operatorname{Exp}(\alpha), G \sim \operatorname{Exp}(\beta)$ and $Z \sim \operatorname{Exp}(\gamma)$. We implement the simulation algorithm for a dynamic contagion process provided by Dassios and Zhao (2011), with parameters set by

$$
\left(a, \lambda_{0}, \rho, \delta ; \alpha, \beta, \gamma ; X_{0}, c\right)=(0.7,0.7,0.5,2.0 ; 2.0,1.5,1.0 ; 10,1.5) .
$$

In Figure 2, we plot the ruin probability $P\left\{\tau^{*}<t \mid X_{0}=x, \lambda_{0}=\lambda\right\}$ against the time from $t=0$ to $t=400$. We can observe that the probability increases and converges around $30 \%$ when time $t$ increases. Note that, each point is calculated based on 50,000 simulated paths of dynamic contagion processes. For instance, one example of simulated surplus process $X_{t}$ with the underlying point process of claim arrival $N_{t}$ and intensity process $\lambda_{t}$ from time $t=0$ to $t=100$ is represented by Figure 3, and the pattern of clustering arrival of claims generated by a dynamic contagion process is also shown in the histogram. For comparison, the theoretical expectations of $\lambda_{t}$ and $N_{t}$ (given by Corollary 2.1) are plotted together with their simulated paths. More numerical examples are provided later by Section 7.3.

\section{Exponential Martingales and Generalised Lundberg's Fundamental Equation}

In this section, we find some useful exponential martingales which link to the generalised Lundberg's fundamental equation. More importantly, they are crucial for deriving some key results of the ruin problem in the later sections. 


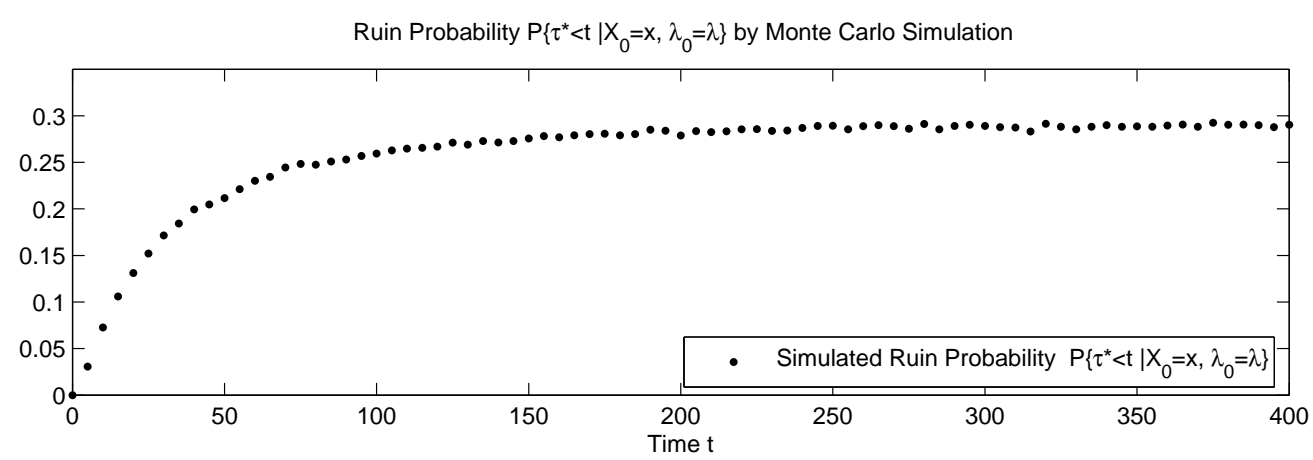

Figure 2: Ruin Probability $P\left\{\tau^{*}<t \mid X_{0}=x, \lambda_{0}=\lambda\right\}$ by 50, 000 Simulated Dynamic Contagion Processes

Theorem 4.1. Assume $\delta>\mu_{1_{G}}$ and the net profit condition (6), we have a martingale

$$
e^{-v_{r} X_{t}} e^{\eta_{r} \lambda_{t}} e^{-r t}, \quad r \geq 0,
$$

where constants $r, v_{r}$ and $\eta_{r}$ satisfy a generalised Lundberg's fundamental equation

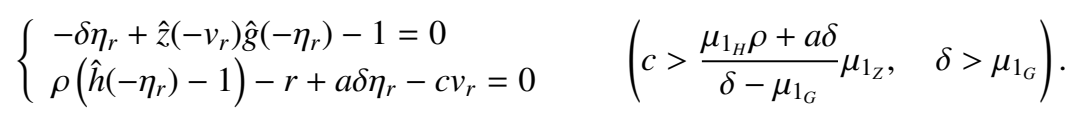

If $0 \leq r<r^{*}$, then (7) has a unique positive solution $\left(v_{r}^{+}>0, \eta_{r}^{+}>0\right)$, where

$$
r^{*}=: \rho\left(\hat{h}\left(-\eta^{*}\right)-1\right)+a \delta \eta^{*}
$$

and $\eta^{*}$ is the unique positive solution to

$$
1+\delta \eta_{r}=\hat{g}\left(-\eta_{r}\right)
$$

Proof. The (Model-1 type) infinitesimal generator of the process $\left(X_{t}, \lambda_{t}, t\right)$ acting on a function $f(x, \lambda, t) \in \Omega(\mathcal{A})$ is given by

$$
\begin{aligned}
\mathcal{A} f(x, \lambda, t)= & \frac{\partial f}{\partial t}-\delta(\lambda-a) \frac{\partial f}{\partial \lambda}+c \frac{\partial f}{\partial x}+\lambda\left(\int_{y=0}^{\infty} \int_{z=0}^{\infty} f(x-z, \lambda+y, t) \mathrm{d} Z(z) \mathrm{d} G(y)-f(x, \lambda, t)\right) \\
& +\rho\left(\int_{0}^{\infty} f(x, \lambda+y, t) \mathrm{d} H(y)-f(x, \lambda, t)\right) .
\end{aligned}
$$

For the classification of Model-1 type and Model-2 type generators for ruin problem, see Dassios and Embrechts (1989).

Assume the form

$$
f(x, \lambda, t)=e^{-v_{r} x} e^{\eta_{r} \lambda} e^{-r t},
$$

and plug into the generator (10). To be a martingale, set $\mathcal{A} f(x, \lambda, t)=0$, then,

$$
-r-\delta(\lambda-a) \eta_{r}-c v_{r}+\lambda\left(\int_{y=0}^{\infty} \int_{z=0}^{\infty} e^{v_{r} z} e^{\eta_{r y} y} \mathrm{~d} Z(z) \mathrm{d} G(y)-1\right)+\rho\left(\int_{0}^{\infty} e^{\eta_{r} y} \mathrm{~d} H(y)-1\right)=0,
$$



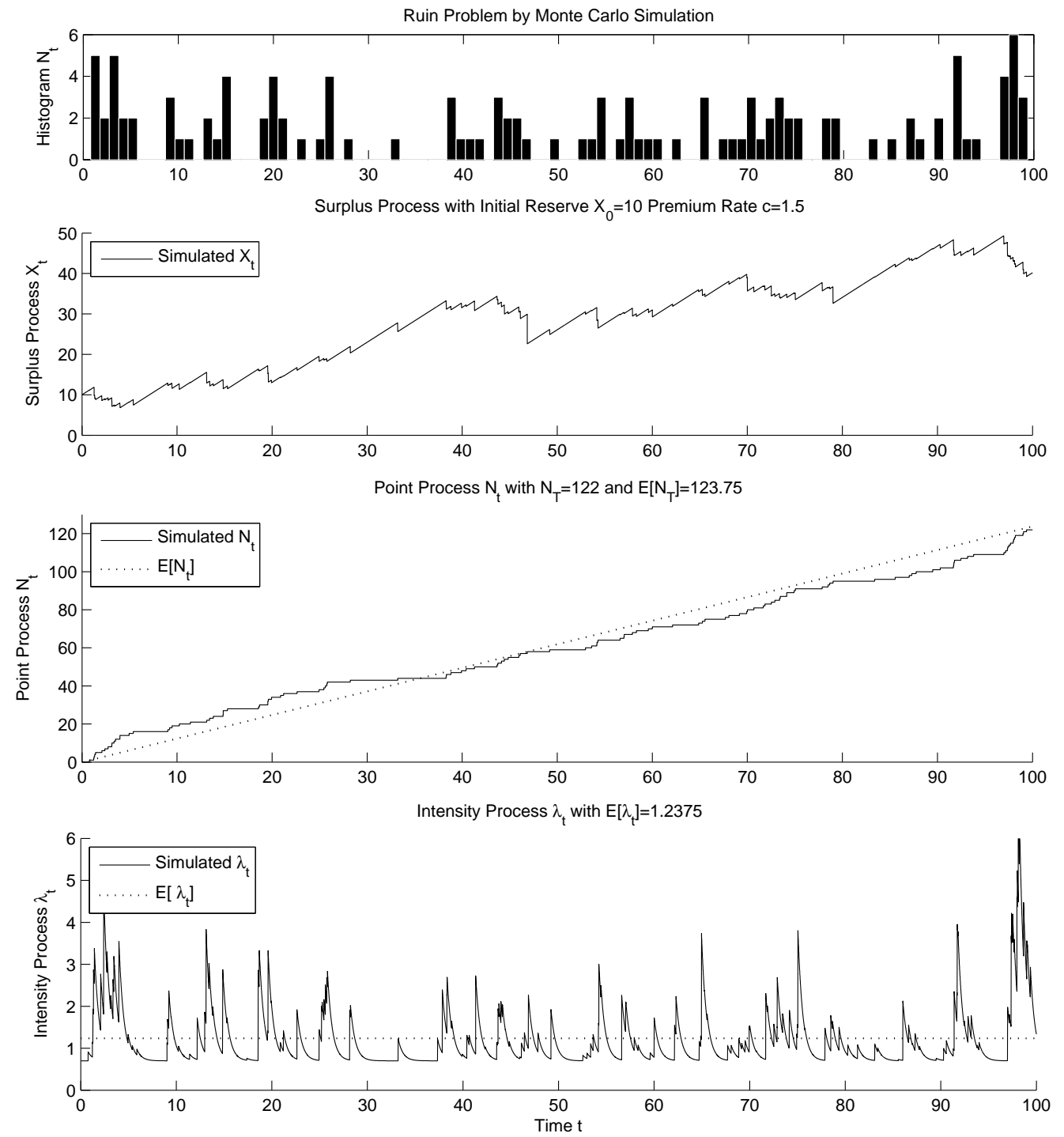

Figure 3: Example: Ruin Problem by One Simulated Dynamic Contagion Process 


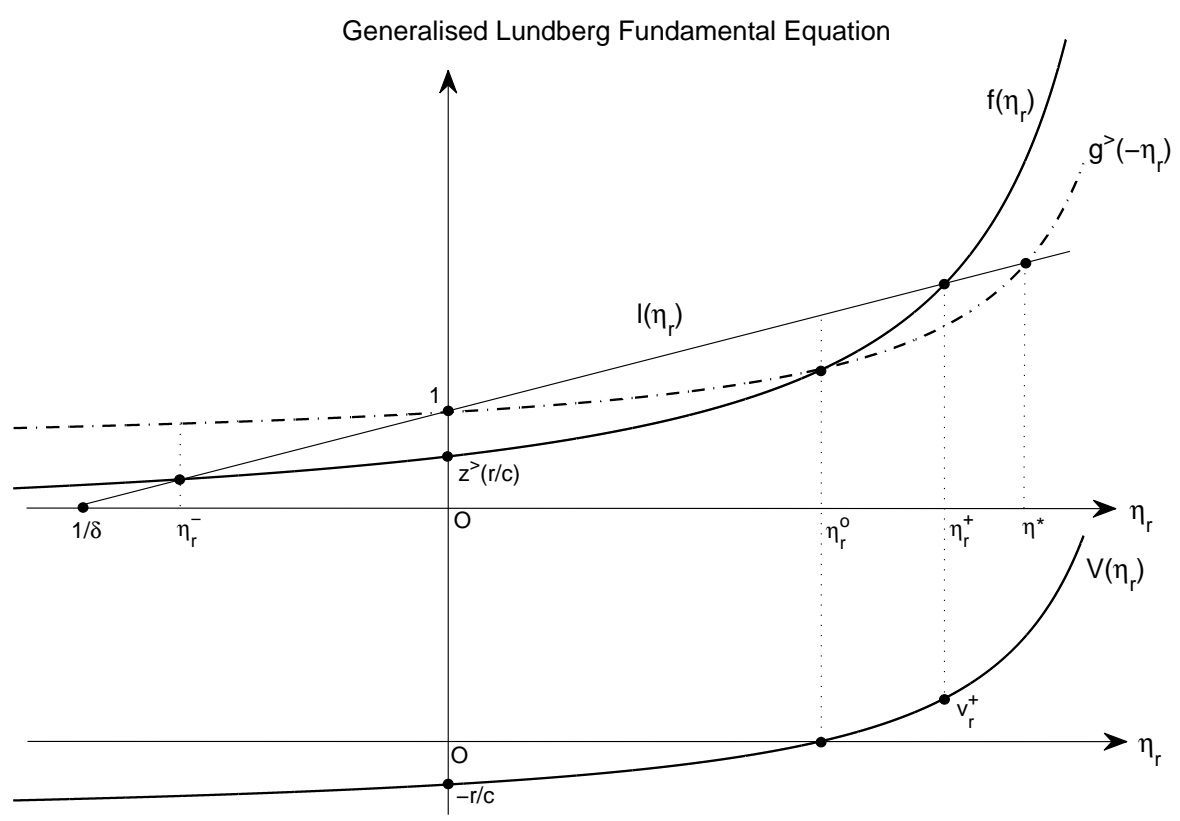

Figure 4: Generalised Lundberg Fundamental Equation

and rewrite as

$$
\left(-\delta \eta_{r}+\hat{z}\left(-v_{r}\right) \hat{g}\left(-\eta_{r}\right)-1\right) \lambda+\left(\rho\left(\hat{h}\left(-\eta_{r}\right)-1\right)-r+a \delta \eta_{r}-c v_{r}\right)=0,
$$

holding for any $\lambda$. Hence, we have (7). The proofs of the uniqueness and the associated conditions for the solution to (7) are given by Lemma 4.1 and Lemma 4.2 as below.

Lemma 4.1. Under $\delta>\mu_{1_{G}}$ and the net profit condition (6), there are unique positive solution $\eta_{r}^{+}$and unique negative solution $\eta_{r}^{-}$to $\eta_{r}$ of the generalised Lundberg's fundamental equation (7); In particular, for $r=0$, there are unique positive solution $\eta_{0}^{+}$and solution zero.

Proof. Rewrite the generalised Lundberg's fundamental equation (7) w.r.t. $\eta_{r}$,

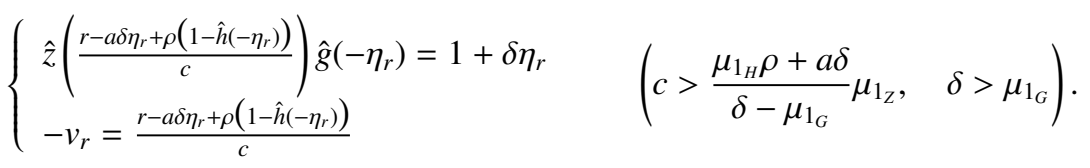

Consider the first equation above, i.e.

$$
f\left(\eta_{r}\right)=l\left(\eta_{r}\right), \quad r>0,
$$

where

$$
\begin{aligned}
f\left(\eta_{r}\right) & =: \quad \hat{z}\left(\frac{r-a \delta \eta_{r}+\rho\left(1-\hat{h}\left(-\eta_{r}\right)\right)}{c}\right) \hat{g}\left(-\eta_{r}\right), \\
l\left(\eta_{r}\right) & =: \quad 1+\delta \eta_{r} .
\end{aligned}
$$


Obviously, $f\left(\eta_{r}\right)$ is a strictly increasing and strictly convex function of $\eta_{r}$, since

$$
\begin{array}{ccc}
\frac{\partial \hat{h}(-u)}{\partial u}>0, & \frac{\partial \hat{g}(-u)}{\partial u}>0, & \frac{\partial \hat{z}(u)}{\partial u}<0, \\
\frac{\partial^{2} \hat{h}(-u)}{\partial u^{2}}>0, & \frac{\partial^{2} \hat{g}(-u)}{\partial u^{2}}>0, & \frac{\partial^{2} \hat{z}(u)}{\partial u^{2}}>0,
\end{array}
$$

and

$$
\begin{aligned}
\frac{\partial f\left(\eta_{r}\right)}{\partial \eta_{r}}= & \left.\frac{-a \delta-\rho \frac{\partial \hat{h}\left(-\eta_{r}\right)}{\partial \eta_{r}}}{c} \frac{\partial \hat{z}(u)}{\partial u}\right|_{u=\frac{r-a \eta_{\eta_{r}+\rho}\left(1-\hat{h}\left(-\eta_{r}\right)\right.}{c}} \hat{g}\left(-\eta_{r}\right) \\
& +\hat{z}\left(\frac{r-a \delta \eta_{r}+\rho\left(1-\hat{h}\left(-\eta_{r}\right)\right)}{c}\right) \frac{\partial \hat{g}\left(-\eta_{r}\right)}{\partial \eta_{r}}>0, \\
\frac{\partial^{2} f\left(\eta_{r}\right)}{\partial \eta_{r}^{2}}= & -\left.\frac{\rho}{c} \frac{\partial^{2} \hat{h}\left(-\eta_{r}\right)}{\partial \eta_{r}^{2}} \frac{\partial \hat{z}(u)}{\partial u}\right|_{u=\frac{r-a \delta \eta_{r}+\rho}{c}\left(1-\hat{h}\left(-\eta_{r}\right)\right)} \hat{g}\left(-\eta_{r}\right) \\
& +\frac{-a \delta-\rho \frac{\partial \hat{h}\left(-\eta_{r}\right)}{\partial \eta_{r}}}{c}\left[\left.\frac{-a \delta-\rho \frac{\partial \hat{h}\left(-\eta_{r}\right)}{\partial \eta_{r}}}{c} \frac{\partial^{2} \hat{z}(u)}{\partial u^{2}}\right|_{u=\frac{r-a \eta_{r}+\rho\left(1-\hat{k}\left(-\eta_{r}\right)\right.}{c}}\right. \\
& \left.+\left.2 \frac{\partial \hat{z}(u)}{\partial u}\right|_{u=\frac{r-a \delta \eta_{r}+\rho\left(1-\hat{h}\left(-\eta_{r}\right)\right)}{c}} \frac{\partial \hat{g}\left(-\eta_{r}\right)}{\partial \eta_{r}}\right] \\
& +\hat{z}\left(\frac{r-a \delta \eta_{r}+\rho\left(1-\hat{h}\left(-\eta_{r}\right)\right)}{c}\right) \frac{\partial^{2} \hat{g}\left(-\eta_{r}\right)}{\partial \eta_{r}^{2}}>0 .
\end{aligned}
$$

Also, $f\left(\eta_{r}\right)>0, f(-\infty)=0, f(+\infty)=+\infty . l\left(\eta_{r}\right)$ is a strictly linearly increasing function of $\eta_{r}$.

We discuss the solutions for the two cases $r>0$ and $r=0$ separately as below.

- For $r>0$, we have

$$
0<f(0)=\hat{z}\left(\frac{r}{c}\right)<1=l(0),
$$

and the slope of the tangent at $\eta_{r}=0$,

$$
\left.\frac{\partial l\left(\eta_{r}\right)}{\partial \eta_{r}}\right|_{\eta_{r}=0}>\left.\frac{\partial f\left(\eta_{r}\right)}{\partial \eta_{r}}\right|_{\eta_{r}=0}>0 .
$$

By the stationarity condition $\delta>\mu_{1_{G}}$ and the net profit condition (6), we have

$$
\begin{aligned}
\left.\frac{\partial f\left(\eta_{r}\right)}{\partial \eta_{r}}\right|_{\eta_{r}=0}= & \left.\frac{-a \delta-\mu_{1_{H}} \rho}{c} \frac{\partial \hat{z}(u)}{\partial u}\right|_{u=\frac{r}{c}}+\hat{z}\left(\frac{r}{c}\right) \mu_{1_{G}} \\
< & \left.\frac{-a \delta-\mu_{1_{H}} \rho}{c} \frac{\partial \hat{z}(u)}{\partial u}\right|_{u=0}+\hat{z}(0) \mu_{1_{G}} \\
& =\frac{a \delta+\mu_{1_{H}} \rho}{c} \mu_{1_{z}}+\mu_{1_{G}} \\
& <\delta=\left.\frac{\partial l\left(\eta_{r}\right)}{\partial \eta_{r}}\right|_{\eta_{r}=0} .
\end{aligned}
$$

It is clear that there are unique positive solution $\eta_{r}^{+}$and unique negative solution $\eta_{r}^{-}$by plotting $f\left(\eta_{r}\right)$ and $l\left(\eta_{r}\right)$, see Figure 4 . 
- For $r=0$, we have

$$
0<f(0)=\hat{z}(0)=1=l(0)
$$

and the slope of the tangent at $\eta_{r}=0$,

$$
\left.\frac{\partial l\left(\eta_{r}\right)}{\partial \eta_{r}}\right|_{\eta_{r}=0}>\left.\frac{\partial f\left(\eta_{r}\right)}{\partial \eta_{r}}\right|_{\eta_{r}=0}>0 .
$$

By the stationarity condition and the net profit condition, we have

$$
\left.\frac{\partial f\left(\eta_{r}\right)}{\partial \eta_{r}}\right|_{\eta_{r}=0}<\frac{a \delta+\mu_{1_{H}} \rho}{c} \mu_{1_{Z}}+\mu_{1_{G}}<\delta=\left.\frac{\partial l\left(\eta_{r}\right)}{\partial \eta_{r}}\right|_{\eta_{r}=0} .
$$

It is clear that there are unique positive solution $\eta_{0}^{+}$and solution 0 by plotting $f\left(\eta_{r}\right)$ and $l\left(\eta_{r}\right)$.

In order to find the positive solution to $v_{r}$, we will only consider the unique positive solution $\eta_{r}^{+}$for $r \geq 0$ in the sequel.

Lemma 4.2. If $0 \leq r<r^{*}$,

$$
r^{*}=: \rho\left(\hat{h}\left(-\eta^{*}\right)-1\right)+a \delta \eta^{*}
$$

where the constant $\eta^{*}$ is the unique positive solution to

$$
1+\delta \eta_{r}=\hat{g}\left(-\eta_{r}\right), \quad \delta>\mu_{1_{G}},
$$

then, there exists a unique positive solution $v_{r}^{+}$to $v_{r}$ of the generalised Lundberg's fundamental equation (7),

$$
v_{r}^{+}=-\frac{r-a \delta \eta_{r}^{+}+\rho\left(1-\hat{h}\left(-\eta_{r}^{+}\right)\right)}{c} .
$$

Proof. By substituting $\eta_{r}^{+}$(from Lemma 4.1) into the second equation of the generalised Lundberg's fundamental equation (7), we have the solution to $v_{r}$, i.e. (12). Define

$$
V\left(\eta_{r}\right)=:-\frac{r-a \delta \eta_{r}+\rho\left(1-\hat{h}\left(-\eta_{r}\right)\right)}{c} .
$$

Obviously, $V\left(\eta_{r}\right)$ is a strictly increasing and strictly convex function of $\eta_{r}$, as $\frac{\partial V\left(\eta_{r}\right)}{\partial \eta_{r}}>0$ and $\frac{\partial^{2} V\left(\eta_{r}\right)}{\partial \eta_{r}^{2}}>0$; also, $V(-\infty)=-\infty, V(+\infty)=+\infty ; v(0)=-\frac{r}{c}<0$; hence, there is unique (positive) root $\eta_{r}^{o}>0$ such that $V\left(\eta_{r}^{o}\right)=0$, also see Figure 4 .

In order to find the unique positive solution $v_{r}^{+}$, such that $v_{r}^{+}=V\left(\eta_{r}^{+}\right)>0$, we have the condition $\eta_{r}^{+}>\eta_{r}^{o}$, which also is equivalent to the condition

$$
l\left(\eta_{r}^{o}\right)>f\left(\eta_{r}^{o}\right), \quad \eta_{r}^{o}>0
$$

or,

$$
1-\delta \eta_{r}^{o}>\hat{g}\left(-\eta_{r}^{o}\right), \quad \eta_{r}^{o}>0
$$


note that, $f\left(\eta_{r}^{o}\right)=\hat{g}\left(-\eta_{r}^{o}\right)$. Under the stationarity condition $\delta>\mu_{1_{G}}$, the equation $1+\delta \eta_{r}=\hat{g}\left(-\eta_{r}\right)$ has the unique positive solution $\eta^{*}$ (independent from $r>0$ ) and the solution 0 . Therefore, we have the condition

$$
0<\eta_{r}^{o}<\eta^{*},
$$

such that

$$
1+\delta \eta_{r}^{o}>\hat{g}\left(-\eta_{r}^{o}\right), \quad \eta_{r}^{o}>0
$$

We discuss the two cases $r>0$ and $r=0$ separately as below.

- If $r=0$, we have $\eta_{0}^{o}=\left.\eta_{r}^{o}\right|_{r=0}=0$, and it is clear that $\eta_{0}^{+}>\eta_{0}^{o}>0$ holds, therefore, $v_{0}^{+}>0$ exists without any condition.

- If $r>0$, then the condition $\eta^{*}>\eta_{r}^{o}>0$ is also equivalent to the condition $V\left(\eta^{*}\right)>0$ since $V(\cdot)$ is a strictly increasing function, i.e.

$$
V\left(\eta^{*}\right)=-\frac{r-a \delta \eta^{*}+\rho\left(1-\hat{h}\left(-\eta^{*}\right)\right)}{c}>0 .
$$

Hence, we can obtain the upper bound $r^{*}$ for $r>0$ explicitly, i.e. $0<r<r^{*}$, where $r^{*}$ is given by (11), note that, $r^{*}>0$ as $\eta^{*}>0$, also see Figure 4 .

Remark 4.1. Given the existence and uniqueness of solution $\left(\eta_{r}^{+}, v_{r}^{+}\right)$to the generalised Lundberg's fundamental equation (7), we have $\eta^{*}>\eta_{r}^{+}$, since

$$
1+\delta \eta_{r}^{+}=\hat{z}\left(-v_{r}^{+}\right) \hat{g}\left(-\eta_{r}^{+}\right)>\hat{g}\left(-\eta_{r}^{+}\right),
$$

we know that, if $\delta>\mu_{1_{G}}$ the equation $1+\delta \eta_{r}=\hat{g}\left(-\eta_{r}\right)$ has solution 0 and $\eta^{*}>0$, then, $\eta_{r}^{+}$should be between them, i.e. $\eta^{*}>\eta_{r}^{+}>0$, also see Figure 4 . Therefore, we have the full ranking

$$
0<\eta_{r}^{o}<\eta_{r}^{+}<\eta^{*}
$$

Remark 4.2. In particular, for $r=0$, we have a martingale $e^{-v_{0}^{+} X_{t}} e^{\eta_{0}^{+} \lambda_{t}}$, where $\left(v_{0}^{+}, \eta_{0}^{+}\right)$is the unique positive solution to the equations

$$
\left\{\begin{array}{l}
\delta \eta_{0}^{+}=\hat{z}\left(-v_{0}^{+}\right) \hat{g}\left(-\eta_{0}^{+}\right)-1 \\
c v_{0}^{+}=a \delta \eta_{0}^{+}+\rho\left(\hat{h}\left(-\eta_{0}^{+}\right)-1\right)
\end{array} \quad\left(c>\frac{\mu_{1_{H}} \rho+a \delta}{\delta-\mu_{1_{G}}} \mu_{1_{Z}}, \quad \delta>\mu_{1_{G}}\right) .\right.
$$

The martingales and generalised Lundberg's fundamental equation derived in this section are the building blocks of the martingale method and change of measure, two key approaches adopted in the following sections.

\section{Ruin Probability via Original Measure}

Theorem 5.1. The ruin probability conditional on $\lambda_{0}$ and $X_{0}$ is given by

$$
P\left\{\tau^{*}<\infty \mid X_{0}=x, \lambda_{0}=\lambda\right\}=\frac{e^{-v_{0}^{+} x} e^{\eta_{0}^{+} \lambda}}{\mathbb{E}\left[e^{-v_{0}^{+} X_{\tau^{*}}} e^{\eta_{0}^{+} \lambda_{\tau^{*}}} \mid \tau^{*}<\infty ; X_{0}=x, \lambda_{0}=\lambda\right]} .
$$


Proof. By the optional stopping theorem, a bounded martingale stopped at a stopping time is still a martingale. Now we consider the martingale found by Theorem 4.1 stopped at the ruin time, i.e.

$$
e^{-v_{r}^{+} X_{\left(\tau^{*} \wedge t\right)}} e^{\eta_{r}^{+} \lambda_{\left(\tau^{*} \wedge t\right)}} e^{-r\left(\tau^{*} \wedge t\right)}, \quad 0 \leq r<r^{*}
$$

By the martingale property, we have

$$
\mathbb{E}\left[e^{-v_{r}^{+} X_{\left(\tau^{*} \wedge t\right)}} e^{\eta_{r}^{+} \lambda_{\left(\tau^{*} \wedge t\right)}} e^{-r\left(\tau^{*} \wedge t\right)}\right]=\mathbb{E}\left[e^{-v_{r}^{+} X_{\left(\tau^{*} \wedge t\right)}} e^{\eta_{r}^{+} \lambda_{\left(\tau^{*} \wedge t\right)}} e^{-r\left(\tau^{*} \wedge t\right)} \mid X_{0}=x, \lambda_{0}=\lambda\right]=e^{-v_{r}^{+} x} e^{\eta_{r}^{+} \lambda},
$$

and

$$
\mathbb{E}\left[e^{-v_{r}^{+} X_{\tau^{*}}} e^{\eta_{r}^{+} \lambda_{\tau^{*}}} e^{-r \tau^{*}} \mid \tau^{*} \leq t\right] P\left\{\tau^{*} \leq t\right\}+\mathbb{E}\left[e^{-v_{r}^{+} X_{t}} e^{\eta_{r}^{+} \lambda_{t}} e^{-r t} \mid \tau^{*}>t\right] P\left\{\tau^{*}>t\right\}=e^{-v_{r}^{+} x} e^{\eta_{r}^{+} \lambda},
$$

or,

$$
\mathbb{E}\left[e^{-v_{r}^{+} X_{\tau^{*}}} e^{\eta_{r}^{+} \lambda_{\tau^{*}}} e^{-r \tau^{*}} \mid \tau^{*} \leq t\right] P\left\{\tau^{*} \leq t\right\}+e^{-r t} \mathbb{E}\left[e^{-v_{r}^{+} X_{t}} e^{\eta_{r}^{+} \lambda_{t}} \mid \tau^{*}>t\right] P\left\{\tau^{*}>t\right\}=e^{-v_{r}^{+} x} e^{\eta_{r}^{+} \lambda}
$$

where

$$
\mathbb{E}\left[e^{-v_{r}^{+} X_{t}} e^{\eta_{r}^{+} \lambda_{t}} \mid \tau^{*}>t\right] P\left\{\tau^{*}>t\right\}=\mathbb{E}\left[e^{-v_{r}^{+} X_{t}} e^{\eta_{r}^{+} \lambda_{t}} \mathbb{I}\left(\tau^{*}>t\right)\right] \leq \mathbb{E}\left[e^{\eta_{r}^{+} \lambda_{t}}\right]
$$

Note that, by Theorem 2.1, we have

$$
\lim _{t \rightarrow \infty} \mathbb{E}\left[e^{\eta_{r}^{+} \lambda_{t}}\right]=\exp \left(\int_{-\eta_{r}^{+}}^{0} \frac{a \delta u+\rho[1-\hat{h}(u)]}{\delta u+\hat{g}(u)-1} \mathrm{~d} u\right)<\infty,
$$

since by Remark 4.1, for $0<r<r^{*}$, we have $-\eta^{*}<-\eta_{r}^{+}<0$ where $-\eta^{*}$ is the negative singular point of the integrand function above, i.e. the unique negative solution to $\delta u+\hat{g}(u)-1=0$. Hence, for the second term in (14),

$$
\lim _{t \rightarrow \infty} e^{-r t} \mathbb{E}\left[e^{-v_{r}^{+} X_{t}} e^{\eta_{r}^{+} \lambda_{t}} \mid \tau^{*}>t\right] P\left\{\tau^{*}>t\right\}=0 .
$$

Let $t \rightarrow \infty$ in (14), then, $\left\{\tau^{*} \leq t\right\} \rightarrow\left\{\tau^{*}<\infty\right\}$, and

$$
\mathbb{E}\left[e^{-v_{r}^{+} X_{\tau^{*}}} e^{\eta_{r}^{+} \lambda_{\tau^{*}}} e^{-r \tau^{*}} \mid \tau^{*}<\infty\right] P\left\{\tau^{*}<\infty\right\}=e^{-v_{r}^{+} x} e^{\eta_{r}^{+} \lambda}
$$

Let $r \rightarrow 0$, we have

$$
\mathbb{E}\left[e^{-v_{0}^{+} X_{\tau^{*}}} e^{\eta_{0}^{+} \lambda_{\tau^{*}}} \mid \tau^{*}<\infty\right] P\left\{\tau^{*}<\infty\right\}=e^{-v_{0}^{+} x} e^{\eta_{0}^{+} \lambda}
$$

then (13) follows.

Corollary 5.1. If $Z \sim \operatorname{Exp}(\gamma)$, then,

$$
P\left\{\tau^{*}<\infty \mid X_{0}=x, \lambda_{0}=\lambda\right\}=\frac{\gamma-v_{0}^{+}}{\gamma} \frac{e^{\eta_{0}^{+} \lambda} e^{-v_{0}^{+} x}}{\mathbb{E}\left[e^{\eta_{0}^{+} \lambda_{\tau^{*}}} \mid \tau^{*}<\infty ; X_{0}=x, \lambda_{0}=\lambda\right]} .
$$


Proof. If $Z \sim \operatorname{Exp}(\gamma)$, due to the memoryless property of the exponential distribution, the overshoot $-X_{\tau^{*}}>0$ then follows the same exponential distribution, i.e. $-X_{\tau^{*}} \sim \operatorname{Exp}(\gamma)$. Hence, for (13) we have

$$
\mathbb{E}\left[e^{-v_{0}^{+} X_{\tau^{*}}} e^{\eta_{0}^{+} \lambda_{\tau^{*}}} \mid \tau^{*}<\infty\right]=\mathbb{E}\left[e^{-v_{0}^{+} X_{\tau^{*}}}\right] \mathbb{E}\left[e^{\eta_{0}^{+} \lambda_{\tau^{*}}} \mid \tau^{*}<\infty\right]=\frac{\gamma}{\gamma-v_{0}^{+}} \mathbb{E}\left[e^{\eta_{0}^{+} \lambda_{\tau^{*}}} \mid \tau^{*}<\infty\right]
$$

Remark 5.1. Note that, the overshoot $-X_{\tau^{*}}>0, \lambda_{\tau^{*}}>0$, then, $e^{-v_{0}^{+} X_{\tau^{*}}}>1, e^{\eta_{0}^{+} \lambda_{\tau^{*}}}>1$, we have an inequality for the ruin probability,

$$
P\left\{\tau^{*}<\infty \mid X_{0}=x, \lambda_{0}=\lambda\right\}<\frac{e^{\eta_{0}^{+} \lambda} e^{-v_{0}^{+} x}}{\mathbb{E}\left[e^{\eta_{0}^{+} \lambda_{\tau^{*}}} \mid \tau^{*}<\infty ; X_{0}=x, \lambda_{0}=\lambda\right]}<e^{\eta_{0}^{+} \lambda} e^{-v_{0}^{+} x} .
$$

$e^{\eta_{0}^{+} \lambda} e^{-v_{0}^{+} x}$ is a rough up bound of ruin probability, as it could be greater than one when $\lambda_{0}$ is relatively large. In order to obtain a more precise upper bound, it is better to find the distribution property of $\mathbb{E}\left[e^{\eta_{0}^{+}} \lambda_{\tau^{*}} \mid \tau^{*}<\infty\right]$ but it would be not easy.

Example 5.1. If $Z \sim \operatorname{Exp}(\gamma)$, then,

$$
P\left\{\tau^{*}<\infty \mid X_{0}=x, \lambda_{0}=\lambda\right\}<\frac{\gamma}{\gamma-v_{0}^{+}} e^{\eta_{0}^{+} \lambda} e^{-v_{0}^{+} x}
$$

For instance, the comparison between the boundaries and the ruin probability $P\left\{\tau^{*}<\infty \mid X_{0}=10, \lambda_{0}=\lambda\right\}$ simulated by 50,000 sample paths with parameter setting

$$
\left(a ; \rho, \delta ; \alpha, \beta, \gamma ; X_{0}, c\right)=(0.7 ; 0.5,2.0 ; 2.0,1.5,1.0 ; 10,1.5), \quad\left(\eta_{0}^{+}, v_{0}^{+}\right)=(0.0842,0.0932),
$$

\begin{tabular}{|c|c|c|c|c|c|c|c|c|c|c|c|c|}
\hline$\lambda_{0}=\lambda$ & 1 & 2 & 3 & 4 & 5 & 6 & 7 & 8 & 9 & 10 & 11 & 12 \\
\hline$P\left\{\tau^{*}<\infty \mid X_{0}=10, \lambda_{0}=\lambda\right\}$ & 28.83 & 3 & 3430 & 37.34 & 40.01 & 43.46 & 6.67 & 50.45 & 53.34 & $56.83^{c}$ & $50.56 \%$ & $\% \quad 63.66 \%$ \\
\hline Up Bound $e^{\eta_{0}^{+} \lambda_{0}} e^{-v_{0}^{+} X_{0}}$ & 42.84 & 6.60 & 50.69 & 5.15 & 59.99 & 65.26 & 70.99 & 77.23 & 84.01 & $91.39^{c}$ & $99.42 \%$ & $\% \mathbf{1 0 8 . 1 6 \%}$ \\
\hline Up Bound $\frac{\gamma-v_{0}^{+}}{\gamma} e^{\eta_{0}^{+} \lambda_{0}} e^{-v_{0}^{+} X_{0}}$ & 38.84 & 2.26 & 15.97 & 50.01 & 54.40 & 59.18 & 54.38 & 70.03 & 76.18 & $82.88^{\circ}$ & $90.16 \%$ & $\% \quad 98.08 \%$ \\
\hline
\end{tabular}

is given by Table 1 and Figure 5 .

Table 1: Example: The Comparison between the Boundaries and the Simulated Ruin Probability

\section{Ruin Probability via Change of Measure}

In this section, we investigate the ruin probability and asymptotics by change of measure via the martingale derived by Theorem 4.1. We will find that under this new measure the ruin becomes certain, and this makes the simulation more efficient than under the original measure where the ruin is not certain and even rare. Similar ideas of improving simulation of rare events by a change of measure can also be found in Asmussen (1985). 


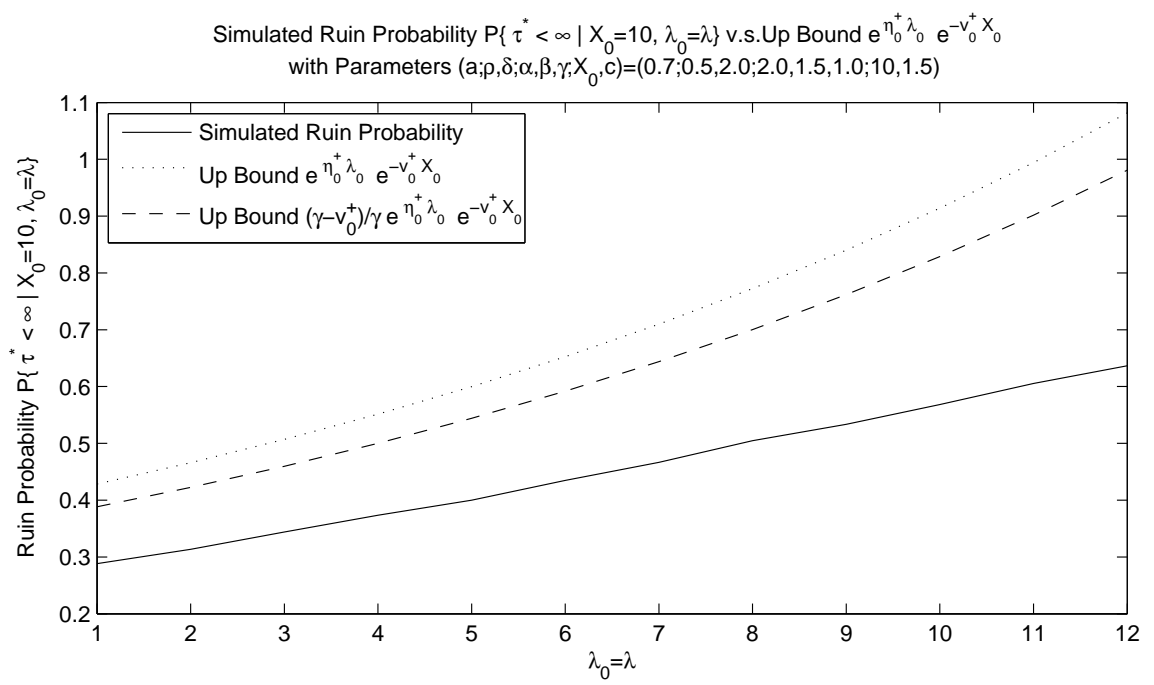

Figure 5: Simulated Ruin Probability $P\left\{\tau^{*}<\infty \mid X_{0}=10, \lambda_{0}=\lambda\right\}$ v.s. Up Bounds

\subsection{Ruin Probability by Change of Measure}

Theorem 6.1. The ruin probability conditional on $X_{0}$ and $\lambda_{0}$ can be expressed under new measure $\widetilde{\mathbb{P}}$ by

$$
P\left\{\tau^{*}<\infty \mid X_{0}=x, \lambda_{0}=\lambda\right\}=e^{-v_{0}^{+} x} e^{m_{0}^{+} \widetilde{\lambda}} \widetilde{\mathbb{E}}\left[\Psi\left(X_{\tau_{-}^{*}}\right) \frac{e^{-m_{0}^{+} \widetilde{\lambda}_{\tau^{*}}}}{\hat{g}\left(-\eta_{0}^{+}\right)} \mid X_{0}=x, \widetilde{\lambda}_{0}=\widetilde{\lambda}\right],
$$

where $\widetilde{\lambda}=:\left(1+\delta \eta_{0}^{+}\right) \lambda, m_{0}^{+}=: \frac{\eta_{0}^{+}}{\delta \eta_{0}^{+}+1}$,

$$
\Psi(x)=: \frac{\bar{Z}(x) e^{v_{0}^{+} x}}{\int_{x}^{\infty} e^{v_{0}^{t} z} \mathrm{~d} Z(z)},
$$

assuming the net profit condition holds under the original measure $\mathbb{P}$, and the stationarity condition holds under both measures $\mathbb{P}$ and $\widetilde{\mathbb{P}}$. The parameter setting for the process $\left(X_{t}, \lambda_{t}\right)$ under $\mathbb{P}$ transforms to the new parameter setting for the process $\left(X_{t}, \widetilde{\lambda_{t}}\right)$ under $\widetilde{\mathbb{P}}$ as follows:

- $a \nearrow \widetilde{a}=:\left(1+\delta \eta_{0}^{+}\right) a$,

- $c \rightarrow \widetilde{c}=: c$,

- $\delta \rightarrow \widetilde{\delta}=: \delta$,

- $\rho \nearrow \widetilde{\rho}=: \hat{h}\left(-\eta_{0}^{+}\right) \rho$,

- $Z(z) \rightarrow \widetilde{Z}(z)$

- $g(u) \rightarrow \widetilde{\widetilde{g}}(u)=: \frac{\vec{g}\left(\frac{u}{1+\delta n_{0}^{+}}\right)}{1+\delta \eta_{0}^{+}}$, 
- $h(u) \rightarrow \widetilde{\bar{h}}(u)=: \frac{\widetilde{h}\left(\frac{u}{1+\delta n_{0}^{+}}\right)}{1+\delta \eta_{0}^{+}}$,

where

$$
\mathrm{d} \widetilde{Z}(z)=: \frac{e^{v_{0}^{+} z} \mathrm{~d} Z(z)}{\hat{z}\left(-v_{0}^{+}\right)}, \quad \mathrm{d} \widetilde{G}(u)=: \frac{e^{\eta_{0}^{+} u} \mathrm{~d} G(u)}{\hat{g}\left(-\eta_{0}^{+}\right)}, \quad \mathrm{d} \widetilde{H}(u)=: \frac{e^{\eta_{0}^{+} u} \mathrm{~d} H(u)}{\hat{h}\left(-\eta_{0}^{+}\right)},
$$

and $\mathrm{d} \widetilde{H}(u)=: \widetilde{h}(u) \mathrm{d} u, \mathrm{~d} \widetilde{G}(u)=: \widetilde{g}(u) \mathrm{d} u ; \mathrm{d} \widetilde{\widetilde{H}}(u)=: \widetilde{\widetilde{h}}(u) \mathrm{d} u, \mathrm{~d} \widetilde{\widetilde{G}}(u)=: \widetilde{\widetilde{g}}(u) \mathrm{d} u$.

Proof. We consider the (Model-2 type) generator

$$
\begin{aligned}
\mathcal{A} f(x, \lambda)= & -\delta(\lambda-a) \frac{\partial f}{\partial \lambda}+c \frac{\partial f}{\partial x}+\lambda\left(\int_{y=0}^{\infty} \int_{z=0}^{x} f(x-z, \lambda+y) \mathrm{d} Z(z) \mathrm{d} G(y)+\bar{Z}(x)-f(x, \lambda)\right) \\
& +\rho\left(\int_{0}^{\infty} f(x, \lambda+y) \mathrm{d} H(y)-f(x, \lambda)\right), \quad x>0 .
\end{aligned}
$$

The solution of the integro-differential equation $\mathcal{A} f(x, \lambda)=0$ is the ruin probability

$$
f(x, \lambda)=P\left\{\tau^{*}<\infty \mid X_{0}=x, \lambda_{0}=\lambda\right\} .
$$

Change Measure from $\mathbb{P}$ to $\widetilde{\mathbb{P}}$. Substitute the function

$$
f(x, \lambda)=e^{-v_{0}^{+} x} e^{\eta_{0}^{+} \lambda} \widetilde{f}(x, \lambda)
$$

into the generator (18), we have

$$
\begin{aligned}
& -\quad \delta(\lambda-a)\left(\eta_{0}^{+} \widetilde{f}+\frac{\partial \widetilde{f}}{\partial \lambda}\right)+c\left(-v_{0}^{+} \widetilde{f}+\frac{\partial \widetilde{f}}{\partial x}\right) \\
& +\lambda\left(\int_{0}^{\infty} \int_{0}^{x} \widetilde{f}(x-z, \lambda+y) e^{v_{0}^{+} z} e^{\eta_{0}^{+} y} \mathrm{~d} Z(z) \mathrm{d} G(y)+\bar{Z}(x) e^{v_{0}^{+} x} e^{-\eta_{0}^{+} \lambda}-\widetilde{f}\right) \\
& +\quad \rho\left(\int_{0}^{\infty} \widetilde{f}(x, \lambda+y) e^{\eta_{0}^{+} y} \mathrm{~d} H(y)-\widetilde{f}\right)=0 .
\end{aligned}
$$

Remind that, by Theorem 4.1 for $r=0$, we have a $\mathcal{F}_{t}^{\mathbb{P}}$-martingale $e^{-v_{0}^{+} X_{t}} e^{\eta_{0}^{+} \lambda_{t}}$ where $\left(v_{0}^{+}, \eta_{0}^{+}\right)$is the unique positive solution to the equations

$$
\left\{\begin{array}{l}
\delta \eta_{0}^{+}=\hat{z}\left(-v_{0}^{+}\right) \hat{g}\left(-\eta_{0}^{+}\right)-1 \\
c v_{0}^{+}=a \delta \eta_{0}^{+}+\rho\left(\hat{h}\left(-\eta_{0}^{+}\right)-1\right)
\end{array} \quad\left(c>\frac{\mu_{1_{H}} \rho+a \delta}{\delta-\mu_{1_{G}}} \mu_{1_{z}}, \quad \delta>\mu_{1_{G}}\right) .\right.
$$

Substitute $c v_{0}^{+}=a \delta \eta_{0}^{+}+\rho\left(\hat{h}\left(-\eta_{0}^{+}\right)-1\right)$ and $\delta \eta_{0}^{+}=\hat{z}\left(-v_{0}^{+}\right) \hat{g}\left(-\eta_{0}^{+}\right)-1$ into (19), we have

$$
\begin{aligned}
& -\delta(\lambda-a) \frac{\partial \widetilde{f}}{\partial \lambda}+c \frac{\partial \widetilde{f}}{\partial x} \\
& +\lambda\left(\int_{0}^{\infty} \int_{0}^{x} \widetilde{f}(x-z, \lambda+y) e^{v_{0}^{+} z} e^{\eta_{0}^{+} y} \mathrm{~d} Z(z) \mathrm{d} G(y)+\bar{Z}(x) e^{\nu_{0}^{+} x} e^{-\eta_{0}^{+} \lambda}-\hat{z}\left(-v_{0}^{+}\right) \hat{g}\left(-\eta_{0}^{+}\right) \hat{f}\right) \\
& +\quad \rho\left(\int_{0}^{\infty} \widetilde{f}(x, \lambda+y) e^{\eta_{0}^{+} y} \mathrm{~d} H(y)-\hat{h}\left(-\eta_{0}^{+}\right) \vec{f}\right)=0 . \\
& 17
\end{aligned}
$$


Change measure (Esscher transform) by (17), and rewrite as

$$
\begin{aligned}
& -\delta(\lambda-a) \frac{\partial \widetilde{f}}{\partial \lambda}+c \frac{\partial \widetilde{f}}{\partial x} \\
& +\hat{z}\left(-v_{0}^{+}\right) \hat{g}\left(-\eta_{0}^{+}\right) \lambda\left(\int_{0}^{\infty} \int_{0}^{x} \widetilde{f}(x-z, \lambda+y) \mathrm{d} \widetilde{Z}(z) \mathrm{d} \widetilde{G}(y)+\bar{Z}(x) \frac{e^{v_{0}^{+} x} e^{-\eta_{0}^{+} \lambda}}{\hat{z}\left(-v_{0}^{+}\right) \hat{g}\left(-\eta_{0}^{+}\right)}-\widetilde{f}\right) \\
& +\hat{h}\left(-\eta_{0}^{+}\right) \rho\left(\int_{0}^{\infty} \widetilde{f}(x, \lambda+y) \mathrm{d} \widetilde{H}(y)-\widetilde{f}\right)=0 .
\end{aligned}
$$

Since $\hat{z}\left(-v_{0}^{+}\right) \hat{g}\left(-\eta_{0}^{+}\right)=1+\delta \eta_{0}^{+}$, we have

$$
\begin{aligned}
& -\delta(\lambda-a) \frac{\partial \widetilde{f}}{\partial \lambda}+c \frac{\partial \widetilde{f}}{\partial x} \\
& +\quad\left(1+\delta \eta_{0}^{+}\right) \lambda\left(\int_{0}^{\infty} \int_{0}^{x} \widetilde{f}(x-z, \lambda+y) \mathrm{d} \widetilde{Z}(z) \mathrm{d} \widetilde{G}(y)+\bar{Z}(x) \frac{e^{v_{0}^{+} x} e^{-\eta_{0}^{+} \lambda}}{\hat{z}\left(-v_{0}^{+}\right) \hat{g}\left(-\eta_{0}^{+}\right)}-\widetilde{f}\right) \\
& +\hat{h}\left(-\eta_{0}^{+}\right) \rho\left(\int_{0}^{\infty} \widetilde{f}(x, \lambda+y) \mathrm{d} \widetilde{H}(y)-\widetilde{f}\right)=0 .
\end{aligned}
$$

Note that,

$$
\overline{\widetilde{Z}}(x)=: \int_{x}^{\infty} \mathrm{d} \widetilde{Z}(z)=\int_{x}^{\infty} \frac{e^{v_{0}^{+} z} \mathrm{~d} Z(z)}{\hat{z}\left(-v_{0}^{+}\right)}=\frac{\int_{x}^{\infty} e^{v_{0}^{+} z} \mathrm{~d} Z(z)}{\hat{z}\left(-v_{0}^{+}\right)},
$$

we have

$$
\bar{Z}(x) \frac{e^{v_{0}^{+} x} e^{-\eta_{0}^{+} \lambda}}{\hat{z}\left(-v_{0}^{+}\right) \hat{g}\left(-\eta_{0}^{+}\right)}=\frac{\bar{Z}(x) e^{v_{0}^{+} x}}{\int_{x}^{\infty} e^{v_{0}^{+} z} \mathrm{~d} Z(z)} \frac{\int_{x}^{\infty} e^{v_{0}^{+} z} \mathrm{~d} Z(z)}{\hat{z}\left(-v_{0}^{+}\right)} \frac{e^{-\eta_{0}^{+} \lambda}}{\hat{g}\left(-\eta_{0}^{+}\right)}=\Psi(x) \frac{e^{-\eta_{0}^{+} \lambda}}{\hat{g}\left(-\eta_{0}^{+}\right)} \overline{\widetilde{Z}}(x),
$$

where $\Psi(x)$ is defined by (16). Hence, we have

$$
\begin{aligned}
& -\quad \delta(\lambda-a) \frac{\partial \widetilde{f}}{\partial \lambda}+c \frac{\partial \widetilde{f}}{\partial x} \\
& +\quad\left(1+\delta \eta_{0}^{+}\right) \lambda\left(\int_{0}^{\infty} \int_{0}^{x} \widetilde{f}(x-z, \lambda+y) \mathrm{d} \widetilde{Z}(z) \mathrm{d} \widetilde{G}(y)+\Psi(x) \frac{e^{-\eta_{0}^{+} \lambda}}{\hat{g}\left(-\eta_{0}^{+}\right)} \widetilde{\widetilde{Z}}(x)-\widetilde{f}\right) \\
& +\quad \hat{h}\left(-\eta_{0}^{+}\right) \rho\left(\int_{0}^{\infty} \widetilde{f}(x, \lambda+y) \mathrm{d} \widetilde{H}(y)-\widetilde{f}\right)=0 .
\end{aligned}
$$

This integro-differential equation has the solution

$$
\widetilde{f}(x, \lambda)=\widetilde{\mathbb{E}}\left[\Psi\left(X_{\tau_{-}^{*}}\right) \frac{e^{-\eta_{0}^{+} \lambda_{\tau_{-}^{*}}}}{\hat{g}\left(-\eta_{0}^{+}\right)} \mathbb{I}\left(\tau^{*}<\infty\right) \mid \lambda_{0}=\lambda, X_{0}=x\right] .
$$

It is similar to the expectation of a Gerber-Shiu penalty function (see Gerber and Shiu (1998)). Therefore, by comparing (20) with (18), we have the parameters for the process $\left(X_{t}, \lambda_{t}\right)$ under $\mathbb{P}$ transformed to the parameters for the process $\left(X_{t}, \lambda_{t}\right)$ under $\widetilde{\mathbb{P}}$ as follows:

- $a \rightarrow \widetilde{a}=a$, 
- $c \rightarrow \widetilde{c}=c$,

- $\delta \rightarrow \widetilde{\delta}=\delta$,

- $\rho \rightarrow \widetilde{\rho}=\hat{h}\left(-\eta_{0}^{+}\right) \rho$,

- $Z(z) \rightarrow \widetilde{Z}(z)$,

- $G(y) \rightarrow \widetilde{G}(y)$,

- $H(y) \rightarrow \widetilde{H}(y)$,

and the ruin probability is given by

$$
P\left\{\tau^{*}<\infty \mid X_{0}=x, \lambda_{0}=\lambda\right\}=e^{-v_{0}^{+} x} e^{\eta_{0}^{+} \lambda} \widetilde{\mathbb{E}}\left[\Psi\left(X_{\tau_{-}^{*}}\right) \frac{e^{-\eta_{0}^{+} \lambda_{\tau_{-}^{*}}}}{\hat{g}\left(-\eta_{0}^{+}\right)} \mathbb{I}\left(\tau^{*}<\infty\right) \mid X_{0}=x, \lambda_{0}=\lambda\right] .
$$

Expression by $\tilde{\lambda}$. Alternatively, we can express the results above w.r.t. $\tilde{\lambda}$ where $\tilde{\lambda}=\left(1+\delta \eta_{0}^{+}\right) \lambda$. Rewrite (20) as

$$
\begin{aligned}
& -\delta\left(\widetilde{\lambda}-\left(1+\delta \eta_{0}^{+}\right) a\right) \frac{\partial \widetilde{f}}{\partial \widetilde{\lambda}}+c \frac{\partial \widetilde{f}}{\partial x} \\
& +\widetilde{\lambda}\left(\int_{0}^{\infty} \int_{0}^{x} \widetilde{f}\left(x-z, \widetilde{\lambda}+\left(1+\delta \eta_{0}^{+}\right) y\right) \mathrm{d} \widetilde{Z}(z) \mathrm{d} \widetilde{G}(y)+\Psi(x) \frac{e^{-\frac{\eta_{0}^{+}}{\partial \eta_{0}^{+}+1}} \bar{g}\left(-\eta_{0}^{+}\right)}{\widetilde{Z}}(x)-\widetilde{f}\right) \\
& +\hat{h}\left(-\eta_{0}^{+}\right) \rho\left(\int_{0}^{\infty} \widetilde{f}\left(x, \widetilde{\lambda}+\left(1+\delta \eta_{0}^{+}\right) y\right) \mathrm{d} \widetilde{H}(y)-\widetilde{f}\right)=0 .
\end{aligned}
$$

Given $\mathrm{d} \widetilde{H}(y)=\widetilde{h}(y) \mathrm{d} y$ and $\mathrm{d} \widetilde{G}(y)=\widetilde{g}(y) \mathrm{d} y$, change variable by $u=\left(1+\delta \eta_{0}^{+}\right) y$, we have the equation of $\widetilde{f}(\widetilde{\lambda}, x)$,

$$
\begin{aligned}
& -\delta\left(\widetilde{\lambda}-\left(1+\delta \eta_{0}^{+}\right) a\right) \frac{\partial \widetilde{f}}{\partial \widetilde{\lambda}}+c \frac{\partial \widetilde{f}}{\partial x} \\
& +\widetilde{\lambda}\left(\int_{0}^{\infty} \int_{0}^{x} \widetilde{f}(x-z, \widetilde{\lambda}+u) \mathrm{d} \widetilde{Z}(z) \frac{\widetilde{g}\left(\frac{u}{1+\delta \eta_{0}^{+}}\right)}{1+\delta \eta_{0}^{+}} \mathrm{d} u+\Psi(x) \frac{e^{-\frac{\eta_{0}^{+}}{\delta \eta_{0}^{+}+1} \widetilde{\lambda}}}{\widehat{g}\left(-\eta_{0}^{+}\right)} \overline{\widetilde{Z}}(x)-\widetilde{f}\right) \\
& +\hat{h}\left(-\eta_{0}^{+}\right) \rho\left(\int_{0}^{\infty} \widetilde{f}(x, \widetilde{\lambda}+u) \frac{\widetilde{h}\left(\frac{u}{1+\delta \eta_{0}^{+}}\right)}{1+\delta \eta_{0}^{+}} \mathrm{d} u-\widetilde{f}\right)=0 .
\end{aligned}
$$

This integro-differential equation has the solution

$$
\widetilde{f}(x, \widetilde{\lambda})=\widetilde{\mathbb{E}}\left[\Psi\left(X_{\tau_{-}^{*}}\right) \frac{e^{-\frac{\eta_{0}^{+}}{\delta \eta_{0}^{+}+1} \widetilde{\lambda}_{\tau_{-}^{*}}}}{\hat{g}\left(-\eta_{0}^{+}\right)} \mathbb{I}\left(\tau^{*}<\infty\right) \mid \lambda_{0}=\lambda, X_{0}=x\right] .
$$

Therefore, by comparing (21) with (18), we have the parameters for the process $\left(X_{t}, \lambda_{t}\right)$ under $\mathbb{P}$ transformed to the parameters for the process $\left(X_{t}, \widetilde{\lambda}_{t}\right)$ under $\widetilde{\mathbb{P}}$ as follows: 
- $a \nearrow \widetilde{a}=\left(1+\delta \eta_{0}^{+}\right) a$,

- $c \rightarrow \widetilde{c}=c$,

- $\delta \rightarrow \widetilde{\delta}=\delta$,

- $\rho \nearrow \widetilde{\rho}=\hat{h}\left(-\eta_{0}^{+}\right) \rho$,

- $Z(z) \rightarrow \widetilde{Z}(z)$,

- $g(u) \rightarrow \widetilde{\widetilde{g}}(u)=\frac{\vec{g}\left(\frac{u}{1+\delta \eta_{0}^{+}}\right)}{1+\delta \eta_{0}^{+}}$,

- $h(u) \rightarrow \widetilde{\widetilde{h}}(u)=\frac{\widetilde{h}\left(\frac{u}{1+\delta \eta_{0}^{+}}\right)}{1+\delta \eta_{0}^{+}}$,

and the ruin probability is given by

$$
\begin{aligned}
& P\left\{\tau^{*}<\infty \mid X_{0}=x, \lambda_{0}=\lambda\right\} \\
= & e^{-v_{0}^{+} x} e^{\eta_{0}^{+} \lambda} \widetilde{\mathbb{E}}\left[\Psi\left(X_{\tau_{-}^{*}}\right) \frac{e^{-m_{0}^{+} \widetilde{\lambda}_{\tau_{-}^{*}}}}{\hat{g}\left(-\eta_{0}^{+}\right)} \mathbb{I}\left(\tau^{*}<\infty\right) \mid X_{0}=x, \lambda_{0}=\lambda\right] \\
= & e^{-v_{0}^{+} x} e^{m_{0}^{+} \bar{\lambda}} \widetilde{\mathbb{E}}\left[\Psi\left(X_{\tau_{-}^{*}}\right) \frac{e^{-m_{0}^{+} \tau_{\tau_{-}^{*}}}}{\hat{g}\left(-\eta_{0}^{+}\right)} \mathbb{I}\left(\tau^{*}<\infty\right) \mid X_{0}=x, \widetilde{\lambda}_{0}=\widetilde{\lambda}\right], \quad m_{0}^{+}=\frac{\eta_{0}^{+}}{\delta \eta_{0}^{+}+1} .
\end{aligned}
$$

By Theorem 6.3 (derived later in this section), if the net profit condition holds under $\mathbb{P}$ and the stationarity condition holds under $\mathbb{P}$ and $\widetilde{\mathbb{P}}$, then the net profit condition can not hold under $\widetilde{\mathbb{P}}$, i.e. $\mathbb{I}\left(\tau^{*}<\infty\right)=1$, hence, we have the ruin probability (15).

Remark 6.1. If $Z \sim \operatorname{Exp}(\gamma)$, then, the expression of the ruin probability (15) can be much simplified, as $\Psi(x)$ is a constant, i.e.

$$
\Psi(x)=\frac{e^{-\gamma x} e^{\nu_{0}^{+} x}}{\int_{x}^{\infty} e^{v_{0} z} \gamma e^{-\gamma z} \mathrm{~d} z}=\frac{\gamma-v_{0}^{+}}{\gamma} .
$$

\subsection{Generalised Cramér-Lundberg Approximation for Exponentially Distributed Claims}

Based on Theorem 6.1, if $Z \sim \operatorname{Exp}(\gamma)$ and the initial intensity follows the stationary distribution under $\widetilde{\mathbb{P}}$, i.e. $\widetilde{\lambda} \sim \Pi$, then, the ruin probability is given by

$$
P\left\{\tau^{*}<\infty \mid X_{0}=x\right\}=\frac{\gamma-v_{0}^{+}}{\gamma \hat{g}\left(-\eta_{0}^{+}\right)} \widetilde{\mathbb{E}}\left[e^{m_{0}^{+} \widetilde{\lambda}}\right] \widetilde{\mathbb{E}}\left[e^{-m_{0}^{+} \widetilde{\lambda}_{\tau_{-}^{*}}} \mid X_{0}=x\right] e^{-v_{0}^{+} x} .
$$

Now, we further generalise the Cramér-Lundberg approximation.

Theorem 6.2. If the claim sizes follows exponential distribution and the initial intensity follows the stationary distribution under $\widetilde{\mathbb{P}}$, i.e. $\widetilde{Z} \sim \operatorname{Exp} \widetilde{\gamma}$ ) and $\widetilde{\lambda} \sim \Pi$, then, the generalised CramérLundberg approximation is given by

$$
P\left\{\tau^{*}<\infty \mid X_{0}=x\right\} \underset{20}{\sim} C e^{-v_{0}^{+} x}, \quad x \rightarrow \infty,
$$


where

$$
C=: \frac{\gamma-v_{0}^{+}}{\gamma \hat{g}\left(-\eta_{0}^{+}\right)} \widetilde{\mathbb{E}}\left[e^{m_{0}^{+} \widetilde{\lambda}}\right] \frac{\frac{1}{\bar{\gamma}} \widetilde{\mathbb{E}}\left[e^{-m_{0}^{+} \widetilde{\lambda}}\right]-\widetilde{c} \widetilde{\mathbb{E}}\left[e^{-m_{0}^{+} \widetilde{\lambda}_{\tau_{-}^{*}}} \mid X_{0}=0\right]}{\frac{1}{\bar{\gamma}} \widetilde{\mathbb{E}}[\widetilde{\lambda}]-\widetilde{c}} .
$$

Proof. Use the new set of parameters under $\widetilde{\mathbb{P}}$ given by Theorem 6.1, and rewrite (21) as

$$
\begin{aligned}
& -\delta(\widetilde{\lambda}-\widetilde{a}) \frac{\partial \widetilde{f}}{\partial \widetilde{\lambda}}+\widetilde{c} \frac{\partial \widetilde{f}}{\partial x} \\
& +\widetilde{\lambda}\left(\int_{0}^{\infty} \int_{0}^{x} \widetilde{f}(x-z, \widetilde{\lambda}+u) \mathrm{d} \widetilde{Z}(z) \mathrm{d} \widetilde{\widetilde{G}}(u)+\Psi(x) \frac{e^{-m_{0}^{+} \tilde{\lambda}}}{\hat{g}\left(-\eta_{0}^{+}\right)} \widetilde{\widetilde{Z}}(x)-\widetilde{f}\right) \\
& +\widetilde{\rho}\left(\int_{0}^{\infty} \widetilde{f}(x, \widetilde{\lambda}+u) \mathrm{d} \widetilde{\widetilde{H}}(u)-\widetilde{f}\right)=0 .
\end{aligned}
$$

If $\widetilde{Z} \sim \operatorname{Exp} \widetilde{\gamma}), \widetilde{\gamma}=\gamma-v_{0}^{+}$under $\widetilde{\mathbb{P}}($ equivalent to $Z \sim \operatorname{Exp}(\gamma)$ under $\mathbb{P})$, then, by Remark 6.1, we have

$$
\begin{aligned}
& -\delta(\tilde{\lambda}-\widetilde{a}) \frac{\partial \widetilde{f}}{\partial \tilde{\lambda}}+\widetilde{c} \frac{\partial \widetilde{f}}{\partial x} \\
& +\widetilde{\lambda}\left(\int_{0}^{\infty} \int_{0}^{x} \widetilde{f}(x-z, \widetilde{\lambda}+u) \widetilde{\gamma} e^{-\widetilde{\gamma} z} \mathrm{~d} z \mathrm{~d} \widetilde{\widetilde{G}}(u)+\frac{\gamma-v_{0}^{+}}{\gamma} \frac{e^{-m_{0}^{+} \tilde{\lambda}}}{\hat{g}\left(-\eta_{0}^{+}\right)} e^{-\widetilde{\gamma} x}-\widetilde{f}\right) \\
& +\widetilde{\rho}\left(\int_{0}^{\infty} \widetilde{f}(x, \widetilde{\lambda}+u) \mathrm{d} \widetilde{\widetilde{H}}(u)-\widetilde{f}\right)=0 .
\end{aligned}
$$

Take Laplace transform w.r.t. $x$, i.e.

$$
\hat{\widetilde{f}}(w, \widetilde{\lambda})=: \mathcal{L}\{\widetilde{f}(x, \widetilde{\lambda})\}=\int_{0}^{\infty} \widetilde{f}(u, \widetilde{\lambda}) e^{-w u} \mathrm{~d} u,
$$

we have

$$
\begin{aligned}
\mathcal{L}\left\{\frac{\partial \widetilde{f}(x, \widetilde{\lambda})}{\partial x}\right\} & =w \hat{\widetilde{f}}(w, \widetilde{\lambda})-\widetilde{f}(0, \tilde{\lambda}), \\
\mathcal{L}\left\{\int_{0}^{x} \widetilde{f}(x-z, \widetilde{\lambda}+u) \widetilde{\gamma} e^{-\widetilde{\gamma} z} \mathrm{~d} z\right\} & =\frac{\widetilde{\gamma}}{\widetilde{\gamma}+w} \tilde{\widetilde{f}}(w, \widetilde{\lambda}+u), \\
\mathcal{L}\left\{e^{-\widetilde{\gamma} x}\right\} & =\frac{1}{\widetilde{\gamma}+w},
\end{aligned}
$$

then,

$$
\begin{aligned}
& -\delta(\widetilde{\lambda}-\widetilde{a}) \frac{\partial \hat{\widetilde{f}}(w, \tilde{\lambda})}{\partial \widetilde{\lambda}}+\widetilde{c}(w \hat{\widetilde{f}}(w, \widetilde{\lambda})-\widetilde{f}(0, \tilde{\lambda})) \\
& +\widetilde{\lambda}\left(\frac{\widetilde{\gamma}}{\widetilde{\gamma}+w} \int_{0}^{\infty} \hat{\widetilde{f}}(w, \widetilde{\lambda}+u) \mathrm{d} \widetilde{\widetilde{G}}(u)+\frac{\gamma-v_{0}^{+}}{\gamma} \frac{e^{-m_{0}^{+} \tilde{\lambda}}}{\hat{g}\left(-\eta_{0}^{+}\right)} \frac{1}{\widetilde{\gamma}+w}-\tilde{\widetilde{f}}(w, \widetilde{\lambda})\right) \\
& +\tilde{\rho}\left(\int_{0}^{\infty} \tilde{\widetilde{f}}(w, \widetilde{\lambda}+u) \mathrm{d} \widetilde{\widetilde{H}}(u)-\hat{\widetilde{f}}(w, \widetilde{\lambda})\right)=0, \\
& 21
\end{aligned}
$$


or,

$\tilde{\mathcal{A}} \hat{\tilde{f}}(w, \tilde{\lambda})+\widetilde{c}(w \hat{\tilde{f}}(w, \widetilde{\lambda})-\widetilde{f}(0, \widetilde{\lambda}))+\widetilde{\lambda}\left(-\frac{w}{\widetilde{\gamma}+w} \int_{0}^{\infty} \tilde{\widetilde{f}}(w, \widetilde{\lambda}+u) \mathrm{d} \widetilde{\widetilde{G}}(u)+\frac{\gamma-v_{0}^{+}}{\gamma} \frac{e^{-m_{0}^{+} \tilde{\lambda}}}{\hat{g}\left(-\eta_{0}^{+}\right)} \frac{1}{\widetilde{\gamma}+w}\right)=0$.

If $\widetilde{\lambda} \sim \Pi$, then,

$\widetilde{\mathbb{E}}\left[\tilde{\mathcal{A}} \tilde{\tilde{f}}(w, \widetilde{\lambda})+\widetilde{c}(w \hat{\widetilde{f}}(w, \widetilde{\lambda})-\widetilde{f}(0, \tilde{\lambda}))+\widetilde{\lambda}\left(-\frac{w}{\widetilde{\gamma}+w} \int_{0}^{\infty} \tilde{\widetilde{f}}(w, \widetilde{\lambda}+u) \mathrm{d} \widetilde{\widetilde{G}}(u)+\frac{\gamma-v_{0}^{+}}{\gamma} \frac{e^{-m_{0}^{+}} \tilde{\lambda}}{\hat{g}\left(-\eta_{0}^{+}\right)} \frac{1}{\widetilde{\gamma}+w}\right)\right]=0$,

and

$\lim _{w \rightarrow 0} \widetilde{\mathbb{E}}\left[\tilde{\mathcal{A}} \hat{\tilde{f}}(w, \widetilde{\lambda})+\widetilde{c}(w \hat{\widetilde{f}}(w, \widetilde{\lambda})-\widetilde{f}(0, \widetilde{\lambda}))+\widetilde{\lambda}\left(-\frac{w}{\widetilde{\gamma}+w} \int_{0}^{\infty} \hat{\tilde{f}}(w, \widetilde{\lambda}+u) \mathrm{d} \widetilde{\widetilde{G}}(u)+\frac{\gamma-v_{0}^{+}}{\gamma} \frac{e^{-m_{0}^{+} \tilde{\lambda}}}{\hat{g}\left(-\eta_{0}^{+}\right)} \frac{1}{\widetilde{\gamma}+w}\right)\right]=0$.

Since

$$
\widetilde{C}=: \lim _{x \rightarrow \infty} \widetilde{f}(x, \widetilde{\lambda})=\lim _{w \rightarrow 0} w \hat{\widetilde{f}}(w, \widetilde{\lambda})
$$

$\lim _{w \rightarrow 0} \frac{w}{\widetilde{\gamma}+w} \int_{0}^{\infty} \hat{\widetilde{f}}(w, \widetilde{\lambda}+u) \mathrm{d} \widetilde{\widetilde{G}}(u)=\int_{0}^{\infty} \lim _{w \rightarrow 0} \frac{w}{\widetilde{\gamma}+w} \hat{\widetilde{f}}(w, \widetilde{\lambda}+u) \mathrm{d} \widetilde{\widetilde{G}}(u)=\int_{0}^{\infty} \frac{1}{\bar{\gamma}} \widetilde{C} \mathrm{~d} \widetilde{\widetilde{G}}(u)=\frac{\widetilde{C}}{\bar{\gamma}}$,

and by Theorem 2.2, we also have $\mathbb{E}[\widetilde{\mathcal{A}} \tilde{\tilde{f}}(0, \tilde{\lambda})]=0$, then,

$$
\widetilde{\mathbb{E}}\left[\widetilde{c}(\widetilde{C}-\widetilde{f}(0, \widetilde{\lambda}))+\widetilde{\lambda}\left(-\frac{\widetilde{C}}{\widetilde{\gamma}}+\frac{\gamma-v_{0}^{+}}{\gamma} \frac{e^{-m_{0}^{+}} \tilde{\lambda}}{\hat{g}\left(-\eta_{0}^{+}\right)} \frac{1}{\widetilde{\gamma}}\right)\right]=0,
$$

and

$$
\widetilde{C}=\frac{\gamma-v_{0}^{+}}{\gamma \hat{g}\left(-\eta_{0}^{+}\right)} \frac{\frac{1}{\tilde{\gamma}} \widetilde{\mathbb{E}}\left[e^{-m_{0}^{+} \widetilde{\lambda}} \widetilde{\lambda}\right]-\widetilde{c} \widetilde{\mathbb{E}}\left[e^{-m_{0}^{+} \tilde{\lambda}_{\tau_{-}^{*}}} \mid \widetilde{\lambda}_{0}=\widetilde{\lambda} \sim \Pi, X_{0}=0\right]}{\frac{1}{\bar{\gamma}} \widetilde{\mathbb{E}}[\widetilde{\lambda}]-\widetilde{c}},
$$

note that, by definition,

$$
\widetilde{\mathbb{E}}[\widetilde{f}(0, \widetilde{\lambda})]=\frac{\gamma-v_{0}^{+}}{\gamma \hat{g}\left(-\eta_{0}^{+}\right)} \widetilde{\mathbb{E}}\left[e^{-m_{0}^{+} \widetilde{\lambda}_{\tau_{-}^{*}}} \mid \widetilde{\lambda}_{0}=\widetilde{\lambda} \sim \Pi, X_{0}=0\right] .
$$

Hence, we have the generalised Cramér-Lundberg constant (22) for $\widetilde{\lambda} \sim \Pi$, as

$$
C=: \lim _{x \rightarrow \infty} \frac{P\left\{\tau^{*}<\infty \mid X_{0}=x\right\}}{e^{-v_{0} x}}=\lim _{x \rightarrow \infty} \widetilde{\mathbb{E}}\left[e^{m_{0}^{+} \tilde{\lambda}} \widetilde{f}(x, \widetilde{\lambda})\right]=\widetilde{\mathbb{E}}\left[e^{m_{0}^{+} \widetilde{\lambda}}\right] \widetilde{C} .
$$

Remark 6.2. For the Cramér-Lundberg constant (22), by Theorem 2.1 and Corollary 2.1, we 
can explicitly calculate the terms

$$
\begin{aligned}
& \widetilde{\mathbb{E}}[\widetilde{\lambda}]=\frac{\mu_{1_{\tilde{H}}} \widetilde{\rho}+\widetilde{a} \widetilde{\delta}}{\widetilde{\delta}-\mu_{1_{\widetilde{G}}}} \\
& \widetilde{\mathbb{E}}\left[e^{m_{0}^{+}} \tilde{\lambda}\right]=\exp \left(\int_{-m_{0}^{+}}^{0} \frac{\widetilde{a} \delta u+\widetilde{\rho}[1-\hat{\widetilde{h}}(u)]}{\widetilde{\delta} u+\hat{\widetilde{g}}(u)-1} \mathrm{~d} u\right) \\
& \widetilde{\mathbb{E}}\left[\widetilde{\lambda} e^{-m_{0}^{+} \widetilde{\lambda}}\right]=-\left.\frac{\mathrm{d}}{\mathrm{d} m} \widetilde{\mathbb{E}}\left[e^{-m \widetilde{\lambda}}\right]\right|_{m=m_{0}^{+}}=\frac{\widetilde{a} \tilde{\delta} m_{0}^{+}+\widetilde{\rho}\left[1-\hat{\widetilde{\widetilde{h}}}\left(m_{0}^{+}\right)\right]}{\widetilde{\delta} m_{0}^{+}+\hat{\widetilde{\widetilde{g}}}\left(m_{0}^{+}\right)-1} \exp \left(-\int_{0}^{m_{0}^{+}} \frac{\widetilde{a} \tilde{\delta} u+\widetilde{\rho}[1-\hat{\tilde{\widetilde{h}}}(u)]}{\widetilde{\delta} u+\hat{\widetilde{g}}(u)-1} \mathrm{~d} u\right) .
\end{aligned}
$$

Also, by Theorem 6.3 for the net profit condition under the measure $\widetilde{\mathbb{P}}$, we have

$$
\frac{1}{\widetilde{\gamma}} \widetilde{\mathbb{E}}[\widetilde{\lambda}]-\widetilde{c}>0
$$

\subsection{Net Profit Condition under $\mathbb{P}$ and $\widetilde{\mathbb{P}}$}

Theorem 6.3. If the net profit condition and the stationarity condition both hold under $\mathbb{P}$, i.e.

$$
c>\frac{\mu_{1_{H}} \rho+a \delta}{\delta-\mu_{1_{G}}} \mu_{1_{Z}}, \quad \delta>\mu_{1_{G}},
$$

and the stationarity condition also holds under the new measure $\widetilde{\mathbb{P}}$, i.e. $\widetilde{\delta}>\mu_{1_{\tilde{\widetilde{G}}}}$, then, under $\widetilde{\mathbb{P}}$, we have

$$
\frac{\mu_{1_{\widetilde{H}}} \widetilde{\rho}+\widetilde{a} \widetilde{\delta}}{\widetilde{\delta}-\mu_{1_{\widetilde{G}}}} \mu_{1_{\widetilde{Z}}}>\widetilde{c}
$$

and the ruin becomes certain (almost surely), i.e.

$$
\widetilde{\mathbb{P}}\left\{\tau^{*}<\infty\right\}=: \lim _{t \rightarrow \infty} \widetilde{\mathbb{P}}\left\{\tau^{*} \leq t\right\}=1 .
$$

Proof. By the transformation between two measures from Theorem 6.1, we have

$$
\mu_{1_{\tilde{Z}}}=: \widetilde{\mathbb{E}}\left[Z_{i}\right]=\int_{0}^{\infty} z \mathrm{~d} \widetilde{Z}(z)=\int_{0}^{\infty} z \frac{e^{v_{0}^{+} z} \mathrm{~d} Z(z)}{\hat{z}\left(-v_{0}^{+}\right)}=\frac{1}{\hat{z}\left(-v_{0}^{+}\right)} \int_{0}^{\infty} z e^{v_{0}^{+} z} \mathrm{~d} Z(z)=\frac{\hat{z}^{\prime}\left(-v_{0}^{+}\right)}{\hat{z}\left(-v_{0}^{+}\right)} .
$$

Change variable $y=\frac{1}{1+\delta \eta_{0}^{+}} u$, then,

$$
\begin{aligned}
& \mu_{1_{\widetilde{H}}}=\widetilde{\mathbb{E}}\left[Y^{(1)}\right]=\int_{0}^{\infty} u \frac{\widetilde{h}\left(\frac{u}{1+\delta \eta_{0}^{+}}\right)}{1+\delta \eta_{0}^{+}} \mathrm{d} u=\frac{\int_{0}^{\infty} u e^{\frac{\eta_{0}^{+}}{1+\delta \eta_{0}^{+}} u} h\left(\frac{1}{1+\delta \eta_{0}^{+}} u\right) \mathrm{d} u}{\left(1+\delta \eta_{0}^{+}\right) \hat{h}\left(-\eta_{0}^{+}\right)}=\frac{1+\delta \eta_{0}^{+}}{\hat{h}\left(-\eta_{0}^{+}\right)} \int_{0}^{\infty} y e^{\eta_{0}^{+} y} \mathrm{~d} H(y) ; \\
& \mu_{1_{\widetilde{\widetilde{G}}}}=\widetilde{\mathbb{E}}\left[Y^{(2)}\right]=\frac{1+\delta \eta_{0}^{+}}{\hat{g}\left(-\eta_{0}^{+}\right)} \int_{0}^{\infty} y e^{\eta_{0}^{+} y} \mathrm{~d} G(y)=\hat{z}\left(-v_{0}^{+}\right) \hat{g}^{\prime}\left(-\eta_{0}^{+}\right) . \quad\left(\because \hat{z}\left(-v_{0}^{+}\right) \hat{g}\left(-\eta_{0}^{+}\right)=1+\delta \eta_{0}^{+}\right)
\end{aligned}
$$




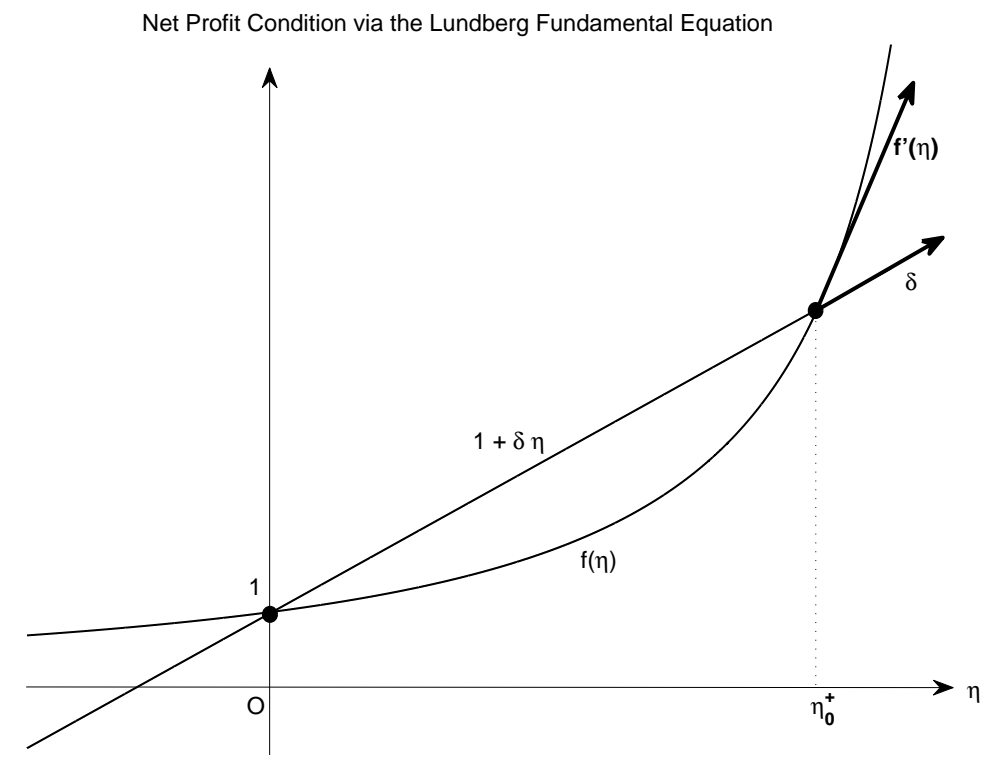

Figure 6: Net Profit Condition via the Generalised Lundberg Fundamental Equation

The mean of self-excited jump sizes under $\widetilde{\mathbb{P}}$ is greater than the one under $\mathbb{P}$, since

$$
\mu_{1_{\widetilde{G}}}>\hat{g}^{\prime}\left(-\eta_{0}^{+}\right)=\int_{0}^{\infty} y e^{\eta_{0}^{+} y} \mathrm{~d} G(y)>\int_{0}^{\infty} y \mathrm{~d} G(y)=\mu_{1_{G}} .
$$

Hence,

$$
\begin{aligned}
& \frac{\mu_{1_{\tilde{H}}} \widetilde{\rho}+\widetilde{a} \tilde{\delta}}{\widetilde{\delta}-\mu_{1_{\widetilde{\widetilde{\sigma}}}}} \mu_{1_{\tilde{z}}} \\
= & \frac{\rho \int_{0}^{\infty} y e^{\eta_{0}^{+} y} \mathrm{~d} H(y)+a \delta}{\delta-\hat{z}\left(-v_{0}^{+}\right) \hat{g}^{\prime}\left(-\eta_{0}^{+}\right)} \frac{1+\delta \eta_{0}^{+}}{\hat{z}\left(-v_{0}^{+}\right)} \int_{0}^{\infty} z e^{v_{0}^{+} z} \mathrm{~d} Z(z) \quad\left(\because \hat{z}\left(-v_{0}^{+}\right) \hat{g}\left(-\eta_{0}^{+}\right)=1+\delta \eta_{0}^{+}\right) \\
= & \hat{z}^{\prime}\left(-v_{0}^{+}\right) \hat{g}\left(-\eta_{0}^{+}\right) \frac{\hat{h}^{\prime}\left(-\eta_{0}^{+}\right) \rho+a \delta}{\delta-\hat{z}\left(-v_{0}^{+}\right) \hat{g}^{\prime}\left(-\eta_{0}^{+}\right)}
\end{aligned}
$$

From the generalised Lundberg's fundamental equation, we have

$$
1+\delta \eta_{0}^{+}=\hat{z}\left(\frac{-a \delta \eta_{0}^{+}+\rho\left(1-\hat{h}\left(-\eta_{0}^{+}\right)\right)}{c}\right) \hat{g}\left(-\eta_{0}^{+}\right) .
$$

If the net profit condition and stationarity condition both holds under $\mathbb{P}$, the right-hand-side function is a strictly increasing and convex function of $\eta_{0}^{+}$as obviously a convex function of a 
function convex function is still a convex function; it was also proved formally in the proof of Lemma 4.1. Hence, as shown in Figure 6, at the point $\eta_{0}^{+}$the slope of the left-hand-side function is greater than the slope of the right-hand-side function, i.e.

$$
\left.\frac{\mathrm{d}}{\mathrm{d} \eta}(1+\delta \eta)\right|_{\eta=\eta_{0}^{+}}<\left.\frac{\mathrm{d}}{\mathrm{d} \eta}\left(\hat{z}\left(\frac{-a \delta \eta+\rho(1-\hat{h}(-\eta))}{c}\right) \hat{g}(-\eta)\right)\right|_{\eta=\eta_{0}^{+}},
$$

or,

$$
\begin{aligned}
\delta< & -\left.\left(\frac{\left.a \delta+\rho \frac{\mathrm{d} \hat{h}\left(-\eta_{0}^{+}\right)}{\mathrm{d} \eta_{0}^{+}}\right)}{c}\right) \frac{\mathrm{d} \hat{z}(u)}{\mathrm{d} u}\right|_{u=\frac{-a \delta \eta_{0}^{+}+\rho\left(1-\hat{h}\left(-\eta_{0}^{+}\right)\right)}{c}} \hat{g}\left(-\eta_{0}^{+}\right)+\hat{z}\left(-v_{0}^{+}\right) \frac{\mathrm{d} \hat{g}\left(-\eta_{0}^{+}\right)}{\mathrm{d} \eta_{0}^{+}} \\
& =-\left.\left(\frac{a \delta+\rho \frac{\mathrm{d} \hat{h}\left(-\eta_{0}^{+}\right)}{\mathrm{d} \eta_{0}^{+}}}{c}\right) \frac{\mathrm{d} \hat{z}(u)}{\mathrm{d} u}\right|_{u=-v_{0}^{+}} \hat{g}\left(-\eta_{0}^{+}\right)+\hat{z}\left(-v_{0}^{+}\right) \frac{\mathrm{d} \hat{g}\left(-\eta_{0}^{+}\right)}{\mathrm{d} \eta_{0}^{+}} \\
& =\left(\frac{a \delta+\rho \frac{\mathrm{d} \hat{h}\left(-\eta_{0}^{+}\right)}{\mathrm{d} \eta_{0}^{+}}}{c}\right) \frac{\mathrm{d} \hat{z}\left(-v_{0}^{+}\right)}{\mathrm{d} v_{0}^{+}} \hat{g}\left(-\eta_{0}^{+}\right)+\hat{z}\left(-v_{0}^{+}\right) \frac{\mathrm{d} \hat{g}\left(-\eta_{0}^{+}\right)}{\mathrm{d} \eta_{0}^{+}},
\end{aligned}
$$

and

$$
c\left(\delta-\hat{z}\left(-v_{0}^{+}\right) \frac{\mathrm{d} \hat{g}\left(-\eta_{0}^{+}\right)}{\mathrm{d} \eta_{0}^{+}}\right)<\left(a \delta+\rho \frac{\mathrm{d} \hat{h}\left(-\eta_{0}^{+}\right)}{\mathrm{d} \eta_{0}^{+}}\right) \frac{\mathrm{d} \hat{z}\left(-v_{0}^{+}\right)}{\mathrm{d} v_{0}^{+}} \hat{g}\left(-\eta_{0}^{+}\right) .
$$

Since the stationarity condition also holds under $\widetilde{\mathbb{P}}$, i.e.

$$
\delta>\hat{z}\left(-v_{0}^{+}\right) \frac{\mathrm{d} \hat{g}\left(-\eta_{0}^{+}\right)}{\mathrm{d} \eta_{0}^{+}}
$$

then,

$$
c<\frac{a \delta+\rho \frac{\mathrm{d} \hat{h}\left(-\eta_{0}^{+}\right)}{\mathrm{d} \eta_{0}^{+}}}{\delta-\hat{z}\left(-v_{0}^{+}\right) \frac{\mathrm{d} \hat{g}\left(-\eta_{0}^{+}\right)}{\mathrm{d} \eta_{0}^{+}}} \hat{g}\left(-\eta_{0}^{+}\right) \frac{\mathrm{d} \hat{z}\left(-v_{0}^{+}\right)}{\mathrm{d} v_{0}^{+}}
$$

and by (25), we have (24).

Remark 6.3. If the net profit condition and the stationarity condition hold under $\mathbb{P}$, but the stationarity condition does not hold under $\widetilde{\mathbb{P}}$, i.e. $\widetilde{\delta}<\mu_{1_{\widetilde{G}}}$, then, the intensity $\widetilde{\lambda}_{t}$ under $\widetilde{\mathbb{P}}$ will increase arbitrarily. It does not mean the measures are not equivalent, as we are only considering them till a fixed time $T$ anyway in the optional stopping theorem; also, ruin does occur with probability one and pretty fast (which will manifest itself in the simulation).

In particular, for the special case of shot noise intensity, interestingly, we find a conjugate relationship between the expected loss rates under the two measures.

Corollary 6.1. For the shot noise case with $H \sim \operatorname{Exp}(\alpha)$ and $Z \sim \operatorname{Exp}(\gamma)$, if the net profit condition holds under the original measure $\mathbb{P}$, i.e.

$$
c>\underset{25}{\frac{\rho}{\delta \alpha \gamma}},
$$


then, under the new measure $\widetilde{\mathbb{P}}$, we have

$$
\widetilde{c}<\frac{\widetilde{\rho}}{\widetilde{\delta \alpha \gamma}}
$$

and

$$
\frac{\rho}{\delta \alpha \gamma} \frac{\widetilde{\rho}}{\widetilde{\delta \alpha \gamma}}=c^{2} .
$$

Proof. In particular, for the shot noise case with jump-size distributions $H \sim \operatorname{Exp}(\alpha)$ and $Z \sim$ $\operatorname{Exp}(\gamma)$ (by setting $a=0$ and $\hat{g}(\cdot)=1$ in Theorem 6.3), we have the parameters transformed by

- $c \rightarrow \widetilde{c}=c$,

- $\delta \rightarrow \widetilde{\delta}=\delta$,

- $\rho \nearrow \widetilde{\rho}=\frac{\alpha}{\alpha-\eta_{0}^{+}} \rho$,

- $\gamma \searrow \widetilde{\gamma}=\gamma-v_{0}^{+}$,

- $\alpha \searrow \widetilde{\alpha}=\frac{\alpha-\eta_{0}^{+}}{1+\delta \eta_{0}^{+}}$,

where the constants are restricted by the generalised Lundberg's fundamental equation

$$
\left\{\begin{array}{l}
\delta \eta_{0}^{+}=\frac{\gamma}{\gamma-v_{0}^{+}}-1 \\
c v_{0}^{+}=\rho\left(\frac{\alpha}{\alpha-\eta_{0}^{+}}-1\right)
\end{array} \quad\left(c>\frac{\rho}{\delta \alpha \gamma}\right) .\right.
$$

The net profit condition holds under $\mathbb{P}$, i.e. $c>\frac{\rho}{\delta \alpha \gamma}$, but under $\widetilde{\mathbb{P}}$ we have $\frac{\widetilde{\rho}}{\delta \widetilde{\alpha \gamma}}>\widetilde{c}$, since

$$
\begin{aligned}
\frac{\widetilde{\rho}}{\widetilde{\delta \widetilde{\alpha}}} & =\frac{\frac{\alpha}{\alpha-\eta_{0}^{+}} \rho}{\frac{\alpha-\eta_{0}^{+}}{1+\delta \eta_{0}^{+}}\left(\gamma-v_{0}^{+}\right) \delta} \\
& =\frac{\alpha \rho}{\delta} \frac{1+\delta \eta_{0}^{+}}{\left(\alpha-\eta_{0}^{+}\right)^{2} \frac{\gamma}{\delta \eta_{0}^{+}+1}} \quad\left(\because \gamma-v_{0}^{+}=\frac{\gamma}{\delta \eta_{0}^{+}+1}\right) \\
& =\frac{\alpha \rho}{\delta \gamma}\left(\frac{1+\delta \eta_{0}^{+}}{\alpha-\eta_{0}^{+}}\right)^{2} \\
& =\frac{\alpha \rho}{\delta \gamma}\left(\frac{c \delta \gamma}{\rho}\right)^{2} \\
& =\frac{\delta \alpha \gamma}{\rho} c^{2} \quad\left(\because c=\frac{1+\delta \eta_{0}^{+}}{\alpha-\eta_{0}^{+}} \frac{\rho}{\delta \gamma}\right) \\
& >\frac{\delta \alpha \gamma}{\rho} \frac{\rho}{\delta \alpha \gamma} c=\widetilde{c} .
\end{aligned}
$$

Hence, we also find (26). 


\section{Example: Jumps with Exponential Distributions}

To represent the previous results in explicit forms, in this section, we further assume the externally excited and self-excited jumps in the intensity process $\lambda_{t}$ and the claim sizes all follow exponential distributions, i.e. $H \sim \operatorname{Exp}(\alpha), G \sim \operatorname{Exp}(\beta)$ and $Z \sim \operatorname{Exp}(\gamma)$, with the density functions

$$
h(y)=\alpha e^{-\alpha y}, \quad g(y)=\beta e^{-\beta y}, \quad z(z)=\gamma e^{-\gamma z}, \quad y, z ; \alpha, \beta, \gamma>0,
$$

and the Laplace transforms

$$
\hat{h}(u)=\frac{\alpha}{\alpha+u}, \quad \hat{g}(u)=\frac{\beta}{\beta+u}, \quad \hat{z}(u)=\frac{\gamma}{\gamma+u} .
$$

\subsection{Generalised Lundberg's Fundamental Equation}

We discuss the general case $0 \leq r<r^{*}$ and the special case $r=0$ for the generalised Lundberg's fundamental equation (from Theorem 4.1) respectively.

Case $0 \leq r<r^{*}$. By Theorem 4.1, we have the generalised Lundberg's fundamental equation for $0 \leq r<r^{*}$,

$$
\left\{\begin{array}{l}
\frac{\gamma}{\gamma-v_{r}} \frac{\beta}{\beta-\eta_{r}}=1+\delta \eta_{r} \\
-v_{r}=\frac{r-a \delta \eta_{r}+\rho\left(1-\frac{\alpha}{\alpha-\eta_{r}}\right)}{c}
\end{array} \quad\left(v_{r}<\gamma, \eta_{r}<(\alpha \wedge \beta) ; c>\frac{\beta(\rho+a \alpha \delta)}{\alpha \gamma(\delta \beta-1)}, \delta \beta>1\right),\right.
$$

or, rewrite it w.r.t. $\eta_{r}$ as

$$
\begin{aligned}
1+\delta \eta_{r} & =\frac{c \gamma \beta\left(\alpha-\eta_{r}\right)}{\left(a \delta \eta_{r}^{2}-(\gamma c+\rho+a \delta \alpha+r) \eta_{r}+\gamma c \alpha+\alpha r\right)\left(\beta-\eta_{r}\right)}, \quad \eta_{r}<(\alpha \wedge \beta), \\
v_{r} & =\frac{\eta_{r}}{c}\left(\frac{\rho}{\alpha-\eta_{r}}+a \delta\right)-\frac{r}{c}, \quad v_{r}<\gamma,
\end{aligned}
$$

with parameters restricted by

$$
c>\frac{\beta(\rho+a \alpha \delta)}{\alpha \gamma(\delta \beta-1)}, \quad \delta \beta>1 .
$$

Solve (9) of Lemma 4.2 and substitute the unique negative solution $\eta^{*}=\frac{\delta \beta-1}{\delta}$ into (8), we obtain the constant $r^{*}$,

$$
r^{*}=(\delta \beta-1)\left(a+\frac{\rho}{\delta(\alpha-\beta)+1}\right) .
$$

Case $r=0$. Set $r \rightarrow 0$, we have the generalised Lundberg's fundamental equation for $r=0$,

$$
\left\{\begin{array}{l}
\frac{\gamma}{\gamma-v_{0}} \frac{\beta}{\beta-\eta_{0}}=1+\delta \eta_{0} \\
-v_{0}=\frac{-a \delta \eta_{0}+\rho\left(1-\frac{\alpha}{\alpha-\eta_{0}}\right)}{c}
\end{array} \quad\left(v_{0}<\gamma, \eta_{0}<(\alpha \wedge \beta) ; c>\frac{\beta(\rho+a \alpha \delta)}{\alpha \gamma(\delta \beta-1)}, \delta \beta>1\right),\right.
$$

or, rewrite w.r.t. $\eta_{0}$ as

$$
\begin{aligned}
1+\delta \eta_{0} & =\frac{c \gamma \beta\left(\alpha-\eta_{0}\right)}{\left(a \delta \eta_{0}^{2}-(\gamma c+\rho+a \delta \alpha) \eta_{0}+\gamma c \alpha\right)\left(\beta-\eta_{0}\right)}, \quad \eta_{0}<(\alpha \wedge \beta), \\
v_{0} & =\frac{\eta_{0}}{c}\left(\frac{\rho}{\alpha-\eta_{0}}+a \delta\right), \quad v_{0}<\gamma,
\end{aligned}
$$


with parameters restricted by

$$
c>\frac{\beta(\rho+a \alpha \delta)}{\alpha \gamma(\delta \beta-1)}, \quad \delta \beta>1 .
$$

The results of case $r=0$ here will be used later in Section 7.3 for numerical calculations.

\subsection{Ruin Probability and Generalised Cramér-Lundberg Approximation via Measure $\widetilde{\mathbb{P}}$}

The Corollary 7.1 below is an example of Theorem 6.1 and Theorem 6.2 by additionally assuming the exponential distributions.

Corollary 7.1. If $H \sim \operatorname{Exp}(\alpha), G \sim \operatorname{Exp}(\beta), Z \sim \operatorname{Exp}(\gamma), \alpha \geq \beta$, the net profit condition holds under $\mathbb{P}$, and stationarity condition holds under $\mathbb{P}$ and $\widetilde{\mathbb{P}}$, and the initial intensity follows the stationary distribution under $\widetilde{\mathbb{P}}$, i.e. $\widetilde{\lambda} \stackrel{\mathcal{D}}{=} \widetilde{a}+\widetilde{\Gamma}_{1}+\widetilde{\Gamma}_{2}$ where

$$
\widetilde{\Gamma}_{1} \sim \operatorname{Gamma}\left(\frac{1}{\bar{\delta}}\left(\widetilde{a}+\frac{\widetilde{\rho}}{\widetilde{\delta}(\widetilde{\alpha}-\widetilde{\beta})+1}\right), \frac{\widetilde{\delta \beta}-1}{\widetilde{\delta}}\right), \quad \widetilde{\Gamma}_{2} \sim \operatorname{Gamma}\left(\frac{\widetilde{\rho}(\widetilde{\alpha}-\widetilde{\beta})}{\widetilde{\delta}(\widetilde{\alpha}-\widetilde{\beta})+1}, \widetilde{\alpha}\right),
$$

then, we have the ruin probability

$$
P\left\{\tau^{*}<\infty \mid X_{0}=x\right\}=\frac{\gamma-v_{0}^{+}}{\gamma} \frac{\beta-\eta_{0}^{+}}{\beta} \widetilde{\mathbb{E}}\left[e^{m_{0}^{+} \widetilde{\lambda}}\right] \widetilde{\mathbb{E}}\left[e^{-m_{0}^{+} \widetilde{\lambda}_{\tau^{*}}} \mid X_{0}=x\right] e^{-v_{0}^{+} x},
$$

and the generalised Cramér-Lundberg approximation

$$
P\left\{\tau^{*}<\infty \mid X_{0}=x\right\} \sim C e^{-v_{0}^{+} x}, \quad x \rightarrow \infty,
$$

where

$$
C=: \frac{\gamma-v_{0}^{+}}{\gamma} \frac{\beta-\eta_{0}^{+}}{\beta} \widetilde{\mathbb{E}}\left[e^{m_{0}^{+} \tilde{\lambda}}\right] \frac{\frac{1}{\tilde{\gamma}} \widetilde{\mathbb{E}}\left[e^{\left.-m_{0}^{+} \widetilde{\lambda} \widetilde{\lambda}\right]}-\widetilde{c \mathbb{E}}\left[e^{-m_{0}^{+} \tilde{\lambda}_{\tau_{-}^{*}}} \mid X_{0}=0\right]\right.}{\frac{1}{\bar{\gamma}} \widetilde{\mathbb{E}}[\widetilde{\lambda}]-\widetilde{c}} .
$$

The transformation from $\mathbb{P}$ to $\widetilde{\mathbb{P}}$ is given by

- $a \nearrow \widetilde{a}=:\left(1+\delta \eta_{0}^{+}\right) a$,

- $c \rightarrow \widetilde{c}=: c$,

- $\delta \rightarrow \widetilde{\delta}=: \delta$,

- $\rho \nearrow \widetilde{\rho}=: \frac{\alpha}{\alpha-\eta_{0}^{+}} \rho$,

- $\gamma \searrow \widetilde{\gamma}=: \gamma-v_{0}^{+}$

- $\beta \searrow \widetilde{\beta}=: \frac{\beta-\eta_{0}^{+}}{1+\delta \eta_{0}^{+}}$,

- $\alpha \searrow \widetilde{\alpha}=: \frac{\alpha-\eta_{0}^{+}}{1+\delta \eta_{0}^{+}}$. 
Proof. If $H \sim \operatorname{Exp}(\alpha), G \sim \operatorname{Exp}(\beta), Z \sim \operatorname{Exp}(\gamma)$, by Theorem 2.3 for the case when $\alpha \geq \beta$, we have the Laplace transform

$$
\widetilde{\mathbb{E}}\left[e^{-m_{0}^{+} \widetilde{\lambda}}\right]=e^{-m_{0}^{+} \widetilde{a}}\left(\frac{\widetilde{\alpha}}{\widetilde{\alpha}+m_{0}^{+}}\right)^{\frac{\tilde{\sigma}(\tilde{\alpha}-\tilde{\beta})}{\delta(\bar{\beta})+1}}\left(\frac{\frac{\widetilde{\delta \beta}-1}{\widetilde{\delta}}}{m_{0}^{+}+\frac{\widetilde{\delta \beta}-1}{\widetilde{\delta}}}\right)^{\frac{1}{\delta}\left(\widetilde{a}+\frac{\tilde{\rho}}{\delta(\tilde{\alpha}-\bar{\beta})+1}\right)} .
$$

Use Theorem 6.1 and Theorem 6.2, the ruin probability and generalised Cramér-Lundberg approximation can be derived immediately.

We only discuss the case when $\alpha \geq \beta$ for instance. It is similar to derive the corresponding results for other cases when $\alpha<\beta$ and we omit them here.

Remark 7.1. We can calculate explicitly for the terms in (27) and (28) of Corollary 7.1,

$$
\begin{aligned}
& \widetilde{\mathbb{E}}\left[e^{m_{0}^{+} \widetilde{\lambda}}\right]=e^{m_{0}^{+} \widetilde{a}}\left(\frac{\widetilde{\alpha}}{\widetilde{\alpha}-m_{0}^{+}}\right)^{\frac{\tilde{\rho}(\tilde{\alpha}-\tilde{\beta})+1}{\delta(\bar{\beta})+1}}\left(\frac{\frac{\widetilde{\delta \beta}-1}{\widetilde{\delta}}}{\frac{\tilde{\delta} \tilde{\beta}-1}{\tilde{\delta}}-m_{0}^{+}}\right)^{\frac{1}{\delta}\left(\widetilde{a}+\frac{\tilde{\sigma}}{\delta(\bar{\alpha}-\tilde{\beta})+1}\right)},
\end{aligned}
$$

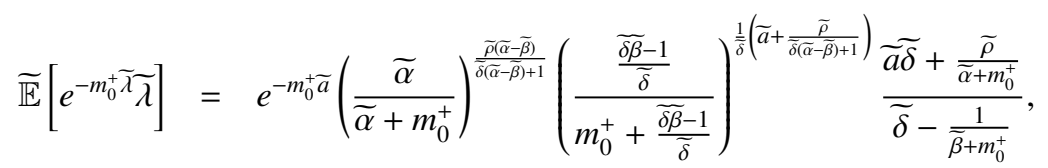

$$
\begin{aligned}
& \widetilde{\mathbb{E}}[\widetilde{\lambda}]=\frac{\widetilde{\underline{\underline{\alpha}}}+\widetilde{a} \widetilde{\delta}}{\widetilde{\delta}-\frac{1}{\bar{\beta}}},
\end{aligned}
$$

except the term $\widetilde{\mathbb{E}}\left[e^{-m_{0}^{+} \tilde{\lambda}_{t_{-}^{*}}} \mid \lambda_{0}=\widetilde{\lambda} \sim \Pi, X_{0}=x\right]$. However, this term can be easily estimated by simulation under $\widetilde{\mathbb{P}}$ where ruin becomes certain. The procedure of estimation is discussed in Section 7.3.

\subsection{Numerical Examples}

For the purpose of simulation, the event of ruin is indicated by comparing the loss with the initial reserve $X_{0}=x$, namely, ruin occurs if

$$
\sup _{t>0}\left\{c t-\sum_{i=i}^{N_{t}} Z_{i}\right\} \geq X_{0} .
$$

Hence, the ruin probability is rewritten as

$$
P\left\{\tau^{*}<\infty \mid X_{0}=x\right\}=P\left\{\sup _{t>0}\left\{c t-\sum_{i=i}^{N_{t}} Z_{i}\right\} \geq x\right\} .
$$

As discussed in Remark 7.1, for exponential distribution case when $\alpha \geq \beta$ of Corollary 7.1, all terms have explicit formulas except the one below that relies on simulation

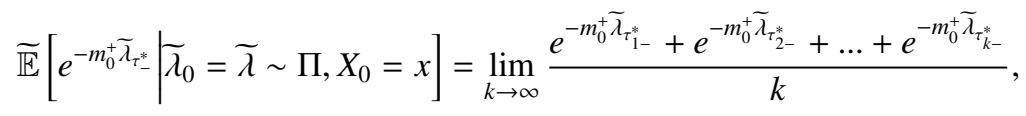

where $k$ is the number of simulations. 
Remark 7.2. Under the original measure $\mathbb{P}$, the event of ruin is rare and particularly it is hard to simulate an infinitely long path $(t=\infty)$ for estimating $P\left\{\tau^{*}<t=\infty \mid X_{0}=x\right\}$ precisely. Thus we alternatively implement the simulation under the measure $\widetilde{\mathbb{P}}$ where ruin becomes certain and hence the simulation is much faster, particularly for $X_{0}=0$.

We provide two numerical examples based on 500, 000 simulations with different parameter settings in Section 7.3.1 and Section 7.3.2, respectively. For each example, we compare the simulated ruin probability and the estimated Cramér constant under $\mathbb{P}$ and $\mathbb{P}$ based on Corollary 7.1.

\subsubsection{Numerical Example 1}

Simulation under $\mathbb{P}$ The parameters under original measure $\mathbb{P}$ are set by

$$
(a, \rho, \delta ; \alpha, \beta, \gamma ; c)=(0.7,0.5,3 ; 2.5,1,1 ; 1.5)
$$

Then, we can obtain $\left(\eta_{0}^{+}, v_{0}^{+}\right)=(0.0811,0.1247)$, the unique solution of the generalised Lundberg's fundamental equation (given by Case $r=0$ of Section 7.1). It is easy to check that $\alpha \geq \beta$, the stationarity condition and the net profit condition all hold, as

$$
\delta=3>\frac{1}{\beta}=1, \quad c=1.5>\frac{\frac{\rho}{\alpha}+a \delta}{\delta-\frac{1}{\beta}} \frac{1}{\gamma}=1.15, \quad \Rightarrow \quad \mathbb{I}\left(\tau^{*}<\infty\right)<1 .
$$

Calculate the ruin probability $P\left\{\tau^{*}<\infty \mid X_{0}=x\right\}$ based on the simulation under $\mathbb{P}$ with $\lambda_{0} \sim \Pi$, i.e. $\lambda_{0} \stackrel{\mathcal{D}}{=} a+\Gamma_{1}+\Gamma_{2}$, where

$$
\Gamma_{1} \sim \operatorname{Gamma}\left(\frac{1}{\delta}\left(a+\frac{\rho}{\delta(\alpha-\beta)+1}\right), \frac{\delta \beta-1}{\delta}\right), \quad \Gamma_{2} \sim \operatorname{Gamma}\left(\frac{\rho(\alpha-\beta)}{\delta(\alpha-\beta)+1}, \alpha\right) .
$$

Since $P\left\{\tau^{*}<\infty \mid X_{0}=x\right\} \sim C e^{-v_{0} x}$, the Cramér constant $C$ can be estimated by the ratio $P\left\{\tau^{*}<\infty \mid X_{0}=x\right\} / e^{-v_{0}^{+} x}$ for a large $X_{0}=x$. The probability $P\left\{\tau^{*}<\infty \mid X_{0}=x\right\}$ and the ratio for $C$ estimation are given by the first and second rows of Table 2 and the plotted by the first and second graphs of Figure 7. Alternatively, it could be more convenient to look at the results by taking logarithm as

$$
\ln \left(P\left\{\tau^{*}<\infty \mid X_{0}=x\right\}\right) \sim \ln C-v_{0} x, \quad x \rightarrow \infty .
$$

\begin{tabular}{|c|c|c|c|c|c|c|c|c|c|c|c|c|c|c|c|c|}
\hline$X_{0}$ & 0 & 2 & 4 & 6 & 8 & 10 & 12 & 14 & 16 & 18 & 20 & 22 & 24 & 26 & 28 & 30 \\
\hline$P\left\{\tau^{*}<\infty \mid X_{0}\right\}$ & $76.90 \%$ & 5656 & & & 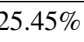 & $19.75^{\circ}$ & & $11.93 \%$ & $9.29 \%$ & $7.24 \%$ & $5.63 \%$ & $4.38 \%$ & $3.42 \%$ & $2.66 \%$ & $2.07 \%$ & $1.62 \%$ \\
\hline$C$ Estimation & $76.90 \%$ & $72.58 \%$ & $70.57 \%$ & $69.59 c$ & $69.03 \%$ & $68.75^{\circ}$ & 68.56 & $68.40 \% 6$ & $68.34 \% 6$ & $68.30 \%$ & $68.14 \% 6$ & $68.14 \%$ & $68.13 \%$ & $68.10 \%$ & 00 & $68.10 \%$ \\
\hline$P_{\widetilde{\mathbb{P}}}\left\{\tau^{*}<\infty \mid X_{0}\right\}$ & $78.71 \%$ & $57.76 \%$ & $43.60 \%$ & $33.42 \%$ & $25.84 \%$ & $20.06 \%$ & $15.56 \%$ & $12.10 \%$ & $9.42 \%$ & $7.33 \%$ & $5.71 \%$ & $4.44 \%$ & $3.47 \%$ & $2.70 \%$ & $2.10 \%$ & $1.64 \%$ \\
\hline $\ln P\left\{\tau^{*}<\infty \mid X_{0}\right\}$ & -0.263 & -0.570 & -0.847 & -1.111 & -1.368 & -1.622 & -1.874 & -2.126 & -2.376 & -2.626 & -2.878 & -3.127 & -3.377 & -3.627 & -3.877 & -4.126 \\
\hline $\ln (66.93 \%)-v_{0} X_{C}$ & $0-0.402$ & -0.651 & -0.900 & -1.150 & -1.399 & -1.649 & -1.898 & -2.148 & -2.397 & -2.647 & -2.896 & -3.145 & -3.395 & -3.644 & -3.894 & -4.143 \\
\hline
\end{tabular}

The results are given by the fourth and fifth rows of Table 2 and plotted by the third graph of Figure 7.

Table 2: Example 1: Numerical Results 

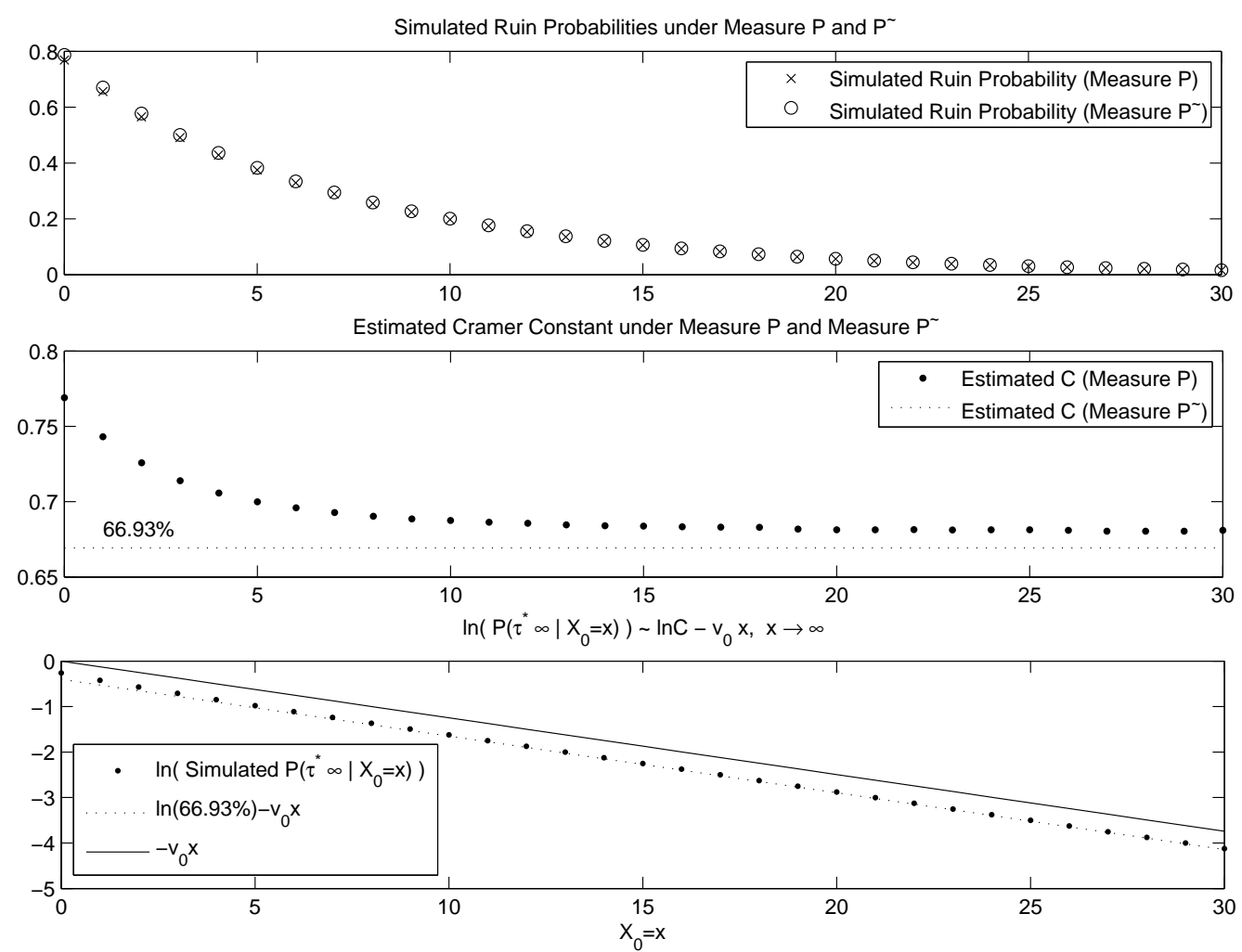

Figure 7: Example 1: Simulated Ruin Probabilities and Estimation for the Cramér Constant $C$ under Measure $\mathbb{P}$ and $\widetilde{\mathbb{P}}$ 
Remark 7.3. The accuracy deceases when $X_{0}=x$ increases, as both of the numerator the ruin probability $P\left\{\tau^{*}<\infty \mid X_{0}=x\right\}$ and the denominator $e^{-v_{0}^{+} x}$ are approaching very closely to 0 . The ruin probability $P\left\{\tau^{*}<\infty \mid X_{0}=x\right\}$ with a high initial reserve becomes harder to be estimated precisely.

Simulation under $\widetilde{\mathbb{P}}$ Under the new measure $\widetilde{\mathbb{P}}$, by the transformation from Corollary 7.1 , the new parameter setting is given by

$$
(\widetilde{a}, \widetilde{\rho}, \widetilde{\delta} ; \widetilde{\alpha}, \widetilde{\beta}, \widetilde{\gamma} ; \widetilde{c})=(0.8703,0.5168,3 ; 1.9455,0.7391,0.8753 ; 1.5000)
$$

Then, we can obtain $m_{0}^{+}=0.0652$. It is easy to check the stationarity condition holds but the net profit condition does not hold and ruin is certain, as

$$
\widetilde{\delta}=3>\frac{1}{\widetilde{\beta}}=1.3530, \quad \widetilde{c}=1.5<\frac{\frac{\widetilde{\rho}}{\tilde{\widetilde{\alpha}}}+\widetilde{a} \tilde{\delta}}{\widetilde{\delta}-\frac{1}{\widetilde{\beta}}} \frac{1}{\bar{\gamma}}=1.9954, \quad \Rightarrow \quad \mathbb{I}\left(\tau^{*}<\infty\right)=1
$$

We can also calculate

$$
\widetilde{\mathbb{E}}\left[e^{m_{0}^{+} \widetilde{\lambda}}\right]=1.1261, \quad \widetilde{\mathbb{E}}\left[e^{-m_{0}^{+} \widetilde{\lambda}} \widetilde{\lambda}\right]=1.4625,
$$

explicitly, and estimate

$$
\widetilde{\mathbb{E}}\left[e^{-m_{0}^{+} \widetilde{\lambda}_{\tau_{-}^{*}}} \mid \widetilde{\lambda}_{0}=\widetilde{\lambda} \sim \Pi, X_{0}=0\right] \approx 86.98 \%
$$

from the simulation under $\widetilde{\mathbb{P}}$ given by third row of Table 3 . Note that, under $\widetilde{\mathbb{P}}$, ruin is certain, i.e. $\widetilde{P}\left\{\tau^{*}<\infty\right\}=1$ and $\widetilde{E}\left[\widetilde{\lambda}_{t}\right]=1.7466$.

Table 3: Example 1: Ruin Simulation under $\widetilde{\mathbb{P}}$ with $X_{0}=0$

\begin{tabular}{ccccccccccccc}
\hline Time $T$ & 5 & 10 & 20 & 40 & 80 & 100 & 150 & 200 & 250 & 300 & 350 & 400 \\
$\widetilde{P}\left\{\left\{\tau^{*}<T\right\}\right.$ & $78.23 \%$ & $87.23 \%$ & $93.22 \%$ & $97.01 \%$ & $99.17 \%$ & $99.44 \%$ & $99.84 \%$ & $99.94 \%$ & $99.98 \%$ & $99.99 \%$ & $100.00 \%$ & $100.00 \%$ \\
$\widetilde{\mathbb{E}}\left[\widetilde{\lambda}_{\pi^{*}}\right]$ & 1.1195 & 1.7354 & 2.5672 & 3.5823 & 4.6133 & 4.8776 & 5.2838 & 5.4970 & 5.4613 & 5.4577 & 5.6493 & 5.5630 \\
$\widetilde{\mathbb{E}}\left[e^{-m_{0}^{+} \widetilde{\lambda}_{\tau^{*}}}\right]$ & 0.8913 & 0.8813 & 0.8767 & 0.8723 & 0.8710 & 0.8709 & 0.8701 & 0.8696 & 0.8709 & 0.8698 & 0.8703 & 0.8699 \\
\hline
\end{tabular}

Hence, $\widetilde{C} \approx 59.4328 \%$ (defined by (23)), and the estimated Cramér constant $C=1.1261 \times$ $59.4328 \%=66.93 \%$, then,

$$
P\left\{\tau^{*}<\infty \mid X_{0}=x\right\} \sim 66.93 \% e^{-0.1247 x}, \quad x \rightarrow \infty .
$$

Here $C$ is consistent with the result (round $68 \%$ in Table 2) obtained earlier by simulation under the original measure $\mathbb{P}$.

The comparison between the ruin probability $P_{\widetilde{\mathbb{P}}}\left\{\tau^{*}<\infty \mid X_{0}\right\}$ (calculated by (27) of Corollary 7.1) simulated under $\widetilde{\mathbb{P}}$ and the ruin probability $P\left\{\tau^{*}<\infty \mid X_{0}\right\}$ simulated directly under $\mathbb{P}$ is given by the first and third rows of Table 2 and the first graph of Figure 7, and the results are very close. 
Remark 7.4. To compare with the classical Poisson model, given $\mathbb{E}\left[\lambda_{t}\right]=1.15$ for the dynamic contagion case in Example 1, we also set the constant intensity $\bar{\lambda}=1.15$ for the Poisson model, and the corresponding Cramér constant can be then calculated exactly by

$$
C=\frac{\bar{\lambda}}{c \gamma}=\frac{1.15}{1.5 \times 1}=76.67 \% \text {. }
$$

\subsubsection{Numerical Example 2}

Similarly, we provide another numerical example by using a different set of parameters. The results are given by Table 4, Table 5, Table 6 and Figure 8.

Remark 7.5. By comparing the simulation of ruin under the original measure $\mathbb{P}$ (given by Figure 2) and under the alternative measure $\widetilde{\mathbb{P}}$ (given by the first row of Table 3 or Table 6), it becomes evident that the simulation is more efficient under $\widetilde{\mathbb{P}}$ as much more events of ruin are realised. For instance, for time $T=100$ in Table 6 , ruin is almost certain as $\widetilde{P}\left\{\tau^{*}<T\right\} \approx 1$.

Table 4: Example 2: Parameters under Measure $\mathbb{P}$ and $\widetilde{\mathbb{P}}$

\begin{tabular}{|c|c|c|c|c|c|c|c|c|c|}
\hline & $a$ & $\rho$ & $\delta$ & $\alpha$ & $\beta$ & $\gamma$ & $c$ & Stationarity & Net Profit \\
\hline Measure $\mathbb{P}$ & 0.7 & 0.5 & 3 & 2 & 1.5 & 1 & 1.5 & Yes & Yes \\
\hline Measure $\mathbb{P}$ & 1.0026 & 0.5388 & 3 & 1.2957 & 0.9467 & 0.7724 & 1.5 & Yes & No \\
\hline & $\eta_{0}^{+}$ & $v_{0}^{+}$ & $m_{0}^{+}$ & $\widetilde{\mathbb{E}}\left[e^{m_{0}^{+}} \tilde{\lambda}\right]$ & $\widetilde{\mathbb{E}}\left[e^{-m_{0}^{+} \widetilde{\lambda}} \widetilde{\lambda}\right]$ & $\widetilde{\mathbb{E}}\left[e^{-m_{0}^{+} \bar{\lambda}_{-}^{*}} \sqrt{\lambda_{0}}=\widetilde{\lambda} \sim \Pi, X_{0}=0\right]$ & $\widetilde{E}\left[\widetilde{\lambda}_{t}\right]$ & $\widetilde{C}$ & $C$ \\
\hline & 0.1441 & 0.2276 & 0.1006 & 1.2019 & 1.3974 & $83.30 \%$ & 1.7615 & $50.06 \%$ & $60.17 \%$ \\
\hline
\end{tabular}

Table 5: Example 2: Numerical Results

\begin{tabular}{|c|c|c|c|c|c|c|c|c|c|c|c|c|c|c|c|c|}
\hline$X_{0}$ & 0 & 2 & 4 & 6 & 8 & 10 & 12 & 14 & 16 & 18 & 20 & 22 & 24 & 26 & 28 & 30 \\
\hline$P\left\{\tau^{*}<\infty \mid X_{0}\right\}$ & $0.05 \%$ & $.52 \%$ & 25720 & .12 & .10 & $.42 \%$ & $4.05 \%$ & $2.58 \%$ & $1.64 \%$ & 1.05 & 0.65 & $0.41 \%$ & $0.26 \%$ & $0.17 \%$ & $0.11 \%$ & $0.07 \%$ \\
\hline$C$ Estimation & $68.65 \%$ & (4) & 05.93 & 0.17 & 02.10 & $02.507 / 00$ & $02.25 \%$ & $02.45 \%$ & 02.4218 & $02.14 / 0$ & סובנים & 02.02 & 02.2010 & $05.25 \%$ & $02.74 \%$ & $62.44 \%$ \\
\hline$P_{\widetilde{\mathbb{P}}}\left\{\tau^{*}<\infty \mid X_{0}\right\}$ & $69.89 \%$ & $41.79 \%$ & $25.76 \%$ & $16.09 \% 1$ & $10.13 \%$ & $6.40 \%$ & $4.05 \%$ & $2.56 \%$ & $1.62 \%$ & $1.03 \%$ & $0.65 \%$ & $0.41 \%$ & $0.26 \%$ & $0.17 \%$ & $0.11 \%$ & $0.07 \%$ \\
\hline $\ln P\left\{\tau^{*}<\infty \mid X_{0}\right\}$ & -0.376 & -0.879 & -1.358 & -1.825 & -2.286 & -2.746 & -3.206 & -3.658 & -4.113 & -4.573 & -5.031 & -5.485 & -5.936 & -6.376 & -6.839 & -7.299 \\
\hline $\mathrm{n}(60.17 \%)-v_{0} X_{0}$ & -0.508 & -0.963 & -1.418 & -1.874 & -2.329 & -2.784 & -3.239 & -3.694 & -4.150 & -4.605 & -5.060 & -5.515 & -5.970 & -6.426 & -6.881 & -7.336 \\
\hline
\end{tabular}

Table 6: Example 2: Ruin Simulation under $\widetilde{\mathbb{P}}$ with $X_{0}=0$

\begin{tabular}{ccccccccccccc}
\hline Time $T$ & 5 & 10 & 20 & 40 & 80 & 100 & 150 & 200 & 250 & 300 & 350 & 400 \\
$\widetilde{P}\left\{\tau^{*}<T\right\}$ & $85.23 \%$ & $92.94 \%$ & $97.50 \%$ & $99.36 \%$ & $99.93 \%$ & $99.97 \%$ & $100.00 \%$ & $100.00 \%$ & $100.00 \%$ & $100.00 \%$ & $100.00 \%$ & $100.00 \%$ \\
$\widetilde{\mathbb{E}}\left[\widetilde{\lambda}_{\tau^{*}}\right]$ & 1.0488 & 1.5517 & 2.1127 & 2.6248 & 2.9161 & 2.9408 & 3.0033 & 2.9541 & 3.0213 & 3.0096 & 2.9674 & 3.0206 \\
$\widetilde{\mathbb{E}}\left[e^{-m \widetilde{\lambda}_{\tau^{*}}}\right]$ & 0.8455 & 0.8396 & 0.8357 & 0.8335 & 0.8332 & 0.8329 & 0.8327 & 0.8330 & 0.8329 & 0.8331 & 0.8331 & 0.8330 \\
\hline
\end{tabular}



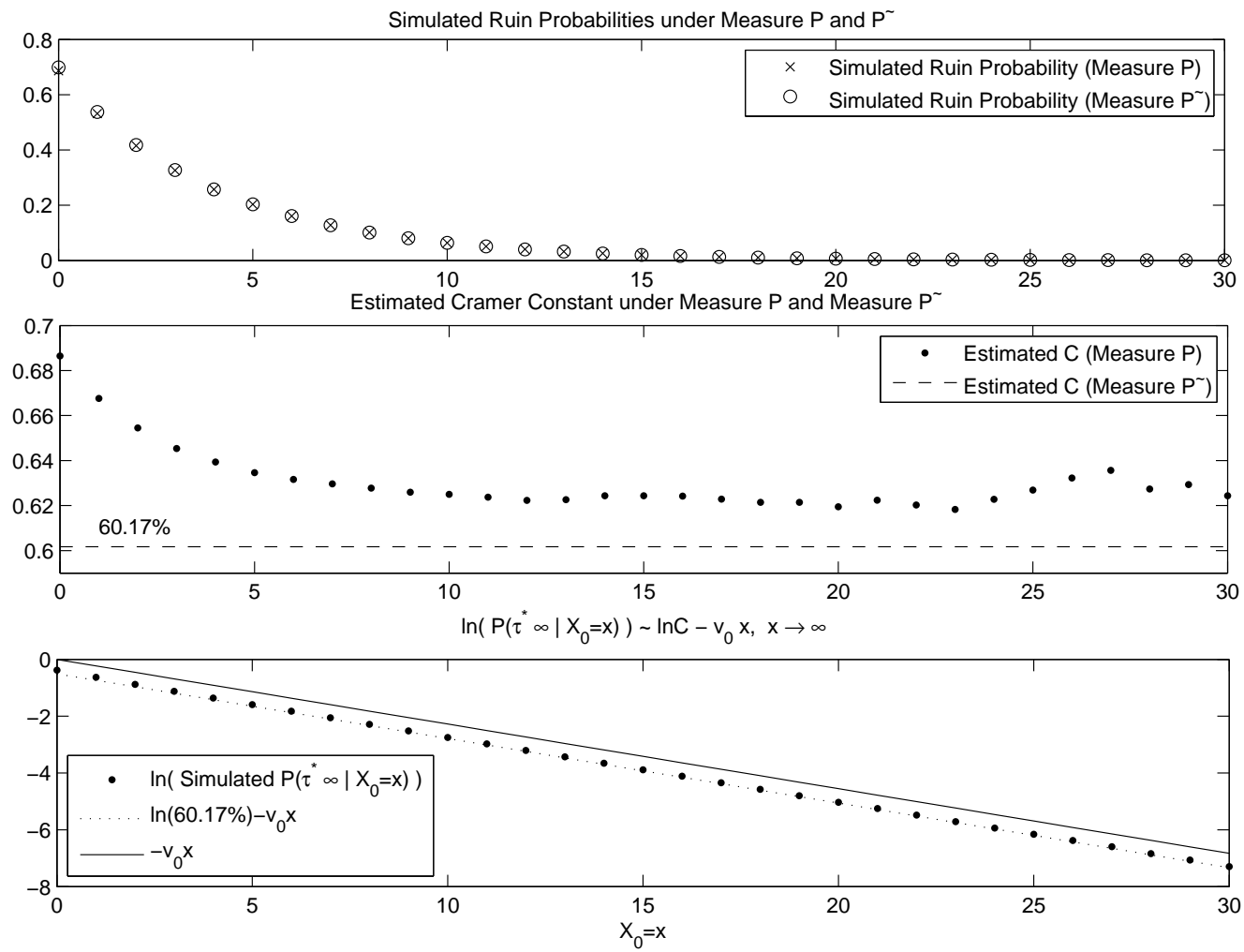

Figure 8: Example 2: Simulated Ruin Probabilities and Estimation for the Cramér Constant $C$ under Measure $\mathbb{P}$ and $\widetilde{\mathbb{P}}$ 


\section{References}

[1] Albrecher, H., Asmussen, S. (2006). Ruin Probabilities and Aggregrate Claims Distributions for Shot Noise Cox Processes. Scandinavian Actuarial Journal. 2: 86-110.

[2] Asmussen, S. (2000). Ruin Probabilities. World Scientific.

[3] Asmussen, S. (1985). Conjugate Processes and the Simulation of Ruin Problems. Stochastic Processes and their Applications . 20: 213-229.

[4] Björk, T., Grandell, J. (1988). Exponential Inequalities for Ruin Probabilities in the Cox Case. Scandinavian Actuarial Journal. 77-111.

[5] Dassios, A., Embrechts, P. (1989). Martingales and Insurance Risk. Communications in Statistics-Stochastic Models. 5(2): 181-217.

[6] Dassios, A., Jang, J. (2003). Pricing of Catastrophe Reinsurance and Derivatives Using the Cox Process with Shot Noise Intensity. Finance $\mathcal{E}$ Stochastics. 7(1): 73-95.

[7] Dassios, A., Zhao, H. (2011). A Dynamic Contagion Process. Advances in Applied Probability. 43(3): 1-33. to appear.

[8] Davis, M.H.A. (1984). Piecewise Deterministic Markov Processes: A General Class of Nondiffusion Stochastic Models. Journal of the Royal Statistical Society B. 46: 353-388.

[9] Davis, M.H.A. (1993). Markov Model and Optimization. Chapman and Hall.

[10] Embrechts, P., Wouters, L. (1990). Simulating Risk Solvency. Insurance: Mathematics and Economics. 9: 141-148.

[11] Embrechts, P., Grandell, J., Schmidli, H. (1993). Finite-time Lundberg Inequalities in the Cox Case. Scandinavian Actuarial Journal. 1: 17-41.

[12] Embrechts, P., Schmidli, H. (1994). Ruin Estimation for a General Insurance Risk Model. Advances in Applied Probability. 26: 404-422.

[13] Gerber, H.U. (1979). An Introduction to Mathematical Risk Theory. Huebner.

[14] Gerber, H.U., Shiu, E.S.W. (1996). Actuarial Bridges to Dynamic Hedging and Option Pricing. Insurance: Mathematics and Economics. 18: 183-218.

[15] Gerber, H.U., Shiu, E.S.W. (1997). The Joint Distribution of the Time of Ruin, the Surplus Immediately before Ruin, and the Deficit at Ruin. Insurance: Mathematics and Economics. 21: 129-137.

[16] Gerber, H.U., Shiu, E.S.W. (1998). On the Time Value of Ruin. North American Actuarial Journal. 2(1): 48-78.

[17] Grandel, J. (1991). Aspects of Risk Theory. Springer.

[18] Hawkes, A.G. (1971). Spectra of Some Self-exciting and Mutually Exciting Point Processes. Biometrika. 58(1), 83-90.

[19] Rolski, T., Schmidli, H., Schmidt, V., Teugels, J. (1999). Stochastic Processes for Insurance and Finance. Wiley, Chichester.

[20] Schmidli, H. (1996). Lundberg Inequalities for a Cox Model with a Piecewise Constant Intensity. Journal of Applied Probability. 33(1): 196-210.

[21] Stabile, G., Torrisi, G.L. (2010). Risk Processes with Non-stationary Hawkes Claims Arrivals. Methodology and Computing in Applied Probability. 12, 415-429. 